\title{
TWISTED GROUP ALGEBRAS SATISFYING A GENERALIZED POLYNOMIAL IDENTITY
}

\author{
D. S. PASSMAN
}

Dedicated to the memory of Professor A. I. Kostrikin

\begin{abstract}
If $K^{t}[G]$ is a twisted group algebra satisfying a nondegenerate multilinear generalized polynomial identity $f\left(\zeta_{1}, \zeta_{2}, \ldots, \zeta_{n}\right)=0$, then we show that $G$ has certain normal subgroups of finite index which can be viewed as being almost central. For example, there exists $H \triangleleft G$ with $|G: H| \cdot\left|H^{\prime}\right|$ bounded by a fixed function of the support sizes of the nonzero $K^{t}[G]$-terms involved in $f$. Indeed, we obtain a more precise version of this result, with the structure of $H$ depending upon the specific twisting in the group algebra. We then go on to determine necessary and sufficient conditions for $K^{t}[G]$ to satisfy such an identity.
\end{abstract}

\section{INTRODUCTION}

Let $K^{t}[G]$ denote a twisted group algebra of the group $G$ over the field $K$. Then $K^{t}[G]$ is a $K$-vector space with basis $\bar{G}$, a copy of $G$. In particular, every element $\alpha \in K^{t}[G]$ can be written uniquely as $\alpha=\sum_{x \in G} k_{x} \bar{x}$, a finite $K$-linear combination of basis elements. Here $k_{x} \in K$, and we use $\operatorname{supp} \alpha=\left\{x \in G \mid k_{x} \neq 0\right\}$ to denote the support of $\alpha$, a finite subset of $G$. In addition, $K^{t}[G]$ is a $K$-algebra and its multiplication is defined distributively by

$$
\bar{x} \bar{y}=t(x, y) \overline{x y} \quad \text { for all } x, y \in G,
$$

where $t: G \times G \rightarrow K^{\bullet}$ is a 2-cocycle, namely a function whose structure is precisely equivalent to the associativity of the algebra. Without loss of generality, we can assume that $\overline{1}=1$ is the identity element of $K^{t}[G]$. Of course, $K^{t}[G]=K[G]$ is the ordinary group algebra of $G$ over $K$ when the twisting function $t(x, y)$ is identically equal to 1.

Now let $R$ be any $K$-algebra. Then a multilinear generalized polynomial over $R$ is a function $f\left(\zeta_{1}, \zeta_{2}, \ldots, \zeta_{n}\right)$ of $n$ noncommuting variables having the form

$$
f\left(\zeta_{1}, \zeta_{2}, \ldots, \zeta_{n}\right)=\sum_{\sigma \in \operatorname{Sym}_{n}} \sum_{j=1}^{a_{\sigma}} \alpha_{0, \sigma, j} \zeta_{\sigma(1)} \alpha_{1, \sigma, j} \zeta_{\sigma(2)} \cdots \alpha_{n-1, \sigma, j} \zeta_{\sigma(n)} \alpha_{n, \sigma, j} .
$$

Here $\operatorname{Sym}_{n}$ is the symmetric group of degree $n$, and each $\alpha_{i, \sigma, j}$ is an element of the ring $R$. Obviously, $f$ is linear in each $\zeta_{i}$, and we say that $R$ satisfies the generalized polynomial identity (g.p.i.) $f$ if

$$
f\left(r_{1}, r_{2}, \ldots, r_{n}\right)=0 \quad \text { for all } r_{1}, r_{2}, \ldots, r_{n} \in R .
$$

2000 Mathematics Subject Classification. 16S34,16R50.

The author's research was supported in part by NSF Grant DMS-9820271. 
Unfortunately, this condition may say nothing about $R$ since, for example, we could have all $\alpha_{i, \sigma, j}=0$. Indeed, even if these terms are nonzero, the polynomial might still be of little interest. As with ordinary polynomial identities, we would like a generalized identity to be indicative of some version of commutativity. To this end, write $f=\sum_{\sigma \in \operatorname{Sym}_{n}} f_{\sigma}$, where

$$
f_{\sigma}\left(\zeta_{1}, \zeta_{2}, \ldots, \zeta_{n}\right)=\sum_{j=1}^{a_{\sigma}} \alpha_{0, \sigma, j} \zeta_{\sigma(1)} \alpha_{1, \sigma, j} \zeta_{\sigma(2)} \cdots \alpha_{n-1, \sigma, j} \zeta_{\sigma(n)} \alpha_{n, \sigma, j} .
$$

Then, we say that $f$ is nondegenerate if there exists some $\tau \in \operatorname{Sym}_{n}$ with $f_{\tau}$ not an identity for $R$. In other words, $f$ is nondegerate if there exists $\tau \in \operatorname{Sym}_{n}$ and suitable elements $s_{1}, s_{2}, \ldots, s_{n} \in R$ with $f_{\tau}\left(s_{1}, s_{2}, \ldots, s_{n}\right) \neq 0$. It follows that if $R$ satisfies a nondegenerate multilinear polynomial identity, then at least two distinct permutations must come into play nontrivially in $f$. By relabeling the variables, if necessary, we can always assume that $f_{1}$ is the nontrivial component of $f$. Note that an ordinary multilinear polynomial is a function of the form

$$
g\left(\zeta_{1}, \zeta_{2}, \ldots, \zeta_{n}\right)=\sum_{\sigma \in \operatorname{Sym}_{n}} \alpha_{\sigma} \zeta_{\sigma(1)} \zeta_{\sigma(2)} \cdots \zeta_{\sigma(n)}
$$

with each $\alpha_{\sigma} \in K$, and $g$ is clearly nondegenerate if and only if $\alpha_{\tau} \neq 0$ for some $\tau$.

Group algebras satisfying an ordinary polynomial identity (p.i.) were classified in [2], [9] and [6], while group algebras satisfying a nondegenerate generalized polynomial identity were handled in [7]. The latter paper also studied p.i. twisted group algebras. In recent years, there has been renewed interest in the g.p.i. result because it turns out to be a key ingredient in the solution of Hartley's problem. Specifically, it was shown in [1] (and then in [3] for finite fields) that if $G$ is periodic and if the unit group of $K[G]$ satisfies a group identity, then $K[G]$ must satisfy a polynomial identity. Furthermore, [4] consided Hartley's problem for twisted group algebras and, in the course of that work, obtained basic properties of twisted group algebras satisfying a g.p.i. In this paper, we sharpen the latter result and prove

Theorem 1.1. Let $K^{t}[G]$ be a twisted group algebra of $G$ over $K$ which satisfies the nondegenerate multilinear generalized polynomial identity

$$
f\left(\zeta_{1}, \zeta_{2}, \ldots, \zeta_{n}\right)=\sum_{\sigma \in \operatorname{Sym}_{n}} \sum_{j=1}^{a_{\sigma}} \alpha_{0, \sigma, j} \zeta_{\sigma(1)} \alpha_{1, \sigma, j} \zeta_{\sigma(2)} \cdots \alpha_{n-1, \sigma, j} \zeta_{\sigma(n)} \alpha_{n, \sigma, j}
$$

with each $\alpha_{i, \sigma, j}$ in $K^{t}[G]$. If

$$
s=\sum_{\sigma \in \mathrm{Sym}_{n}} \sum_{j=1}^{a_{\sigma}} \prod_{i=1}^{n}\left|\operatorname{supp} \alpha_{i, \sigma, j}\right|
$$

then $G$ has a normal subgroup $H$ with $|G: H| \cdot\left|H^{\prime}\right|$ bounded by a function of $s$.

Indeed, we obtain a more precise version of this theorem, with the structure of $H$ depending upon the particular twisting in $K^{t}[G]$. Results of this nature are proved by a coset counting argument, discovered independently in [5] and [9], and known as the Delta Method. As will be apparent, we push this method to the limit and beyond in the following two sections. Specifically, Section 2 is concerned with linear identities in twisted group algebras, namely identities of the form

$$
\alpha_{1} \bar{x} \beta_{1}+\alpha_{2} \bar{x} \beta_{2}+\cdots+\alpha_{n} \bar{x} \beta_{n}=0 \quad \text { for all } x \in T,
$$


where $T$ is a "large" subset of $G$. Section 3 then builds upon this to prove the main theorems on generalized polynomial identities.

At this point, it is appropriate to recall a number of basic definitions, but in a slightly more general context. To start with, for any groups $G \subseteq \mathfrak{G}$, we let

$$
\mathbb{D}=\mathbb{D}_{\mathfrak{G}}(G)=\left\{x \in \mathfrak{G}|| G: \mathbb{C}_{G}(x) \mid<\infty\right\} .
$$

Then $\mathbb{D}$ is a subgroup of $\mathfrak{G}$ normalized by $G$, and it is the set of all elements of $\mathfrak{G}$ having a finite number of $G$-conjugates. Next, for each integer $n \geq 1$, we define

$$
\mathbb{D}_{n}=\mathbb{D}_{n, \mathfrak{G}}(G)=\left\{x \in \mathfrak{G}|| G: \mathbb{C}_{G}(x) \mid \leq n\right\} .
$$

This time $\mathbb{D}_{n}$ is a subset of $\mathfrak{G}$ normalized by $G$, and contained in the group $\mathbb{D}$. Clearly, $\mathbb{D}_{n} \mathbb{D}_{m} \subseteq \mathbb{D}_{n m}$. If $\mathfrak{G}=G$, we usually write $\mathbb{D}_{G}(G)=\Delta(G)$ and $\mathbb{D}_{n, G}(G)=$ $\Delta_{n}(G)$. The characteristic subgroup $\Delta(G)$ is the f.c. (finite conjugate) center of $G$.

Now suppose $K^{t}[\mathfrak{G}]$ is a twisted group algebra and, for each $x \in \mathfrak{G}$, let

$$
\mathbb{C}_{G}^{t}(x)=\{y \in G \mid \bar{x} \bar{y}=\bar{y} \bar{x}\}
$$

denote its twisted centralizer in $G$. Then it is easy to see that $\mathbb{C}_{G}^{t}(x)$ is a subgroup of $G$ contained in the ordinary centralizer $\mathbb{C}_{G}(x)$, and that its index $\left|G: \mathbb{C}_{G}^{t}(x)\right|$ measures the number of distinct conjugates $\bar{x}^{\bar{y}}=\bar{y}^{-1} \bar{x} \bar{y} \in K^{t}[\mathfrak{G}]$ with $y \in G$. There are also twisted versions of $\mathbb{D}$ and $\mathbb{D}_{n}$ given by

$$
\mathbb{D}^{t}=\mathbb{D}_{\mathfrak{G}}^{t}(G)=\left\{x \in \mathfrak{G}|| G: \mathbb{C}_{G}^{t}(x) \mid<\infty\right\}
$$

and

$$
\mathbb{D}_{n}^{t}=\mathbb{D}_{n, \mathfrak{G}}^{t}(G)=\left\{x \in \mathfrak{G}|| G: \mathbb{C}_{G}^{t}(x) \mid \leq n\right\} .
$$

Then $\mathbb{D}^{t}$ is a subgroup of $\mathfrak{G}$, normalized by $G$, and contained in $\mathbb{D}(G)$. Furthermore, $\mathbb{D}_{n}^{t}$ is a subset of $\mathfrak{G}$, normalized by $G$, and contained in $\mathbb{D}_{n}(G)$. Of course, $\mathbb{D}_{n}^{t} \mathbb{D}_{m}^{t} \subseteq$ $\mathbb{D}_{n m}^{t}$, and if $G \triangleleft \mathfrak{G}$ then $\mathbb{D}, \mathbb{D}^{t}, \mathbb{D}_{n}$ and $\mathbb{D}_{n}^{t}$ are normal subsets of $\mathfrak{G}$. When $\mathfrak{G}=G$, we write $\mathbb{D}_{G}^{t}(G)=\Delta^{t}(G)$ and $\mathbb{D}_{n, G}^{t}(G)=\Delta_{n}^{t}(G)$.

Finally, if $S$ is any subset of $\mathfrak{G}$, let $K^{t}[S]$ denote the set of all elements of $K^{t}[\mathfrak{G}]$ with support contained in $S$. For example, if $S=\mathfrak{H}$ is a subgroup of $\mathfrak{G}$, then $K^{t}[\mathfrak{H}]$ is the usual subalgebra of $K^{t}[\mathfrak{G}]$ associated with $\mathfrak{H}$. Now, for any such subset $S$, there is a natural $K$-linear projection map $\pi_{S}: K^{t}[\mathfrak{G}] \rightarrow K^{t}[S] \subseteq K^{t}[\mathfrak{G}]$ given by

$$
\pi_{S}(\bar{x})= \begin{cases}\bar{x}, & \text { if } x \in S, \text { or } \\ 0, & \text { if } x \in \mathfrak{G} \backslash S .\end{cases}
$$

Thus

$$
\pi_{S}: \sum_{x \in \mathfrak{G}} k_{x} \bar{x} \mapsto \sum_{x \in S} k_{x} \bar{x}
$$

and it is easy to see that if $S$ is normalized by $G$, then $\pi_{S}\left(\bar{y}^{-1} \alpha \bar{y}\right)=\bar{y}^{-1} \pi_{S}(\alpha) \bar{y}$ for all $\alpha \in K^{t}[\mathfrak{G}]$ and $y \in G$. For the most part, we are concerned with the special subsets of $\mathfrak{G}$ defined in the preceding paragraphs, and the corresponding projection maps for these sets are denoted by

$$
\phi: K^{t}[\mathfrak{G}] \rightarrow K^{t}[\mathbb{D}], \quad \phi^{t}: K^{t}[\mathfrak{G}] \rightarrow K^{t}\left[\mathbb{D}^{t}\right]
$$

and

$$
\phi_{n}: K^{t}[\mathfrak{G}] \rightarrow K^{t}\left[\mathbb{D}_{n}\right], \quad \phi_{n}^{t}: K^{t}[\mathfrak{G}] \rightarrow K^{t}\left[\mathbb{D}_{n}^{t}\right] .
$$

Since $\mathbb{D}$ and $\mathbb{D}^{t}$ are subgroups of $\mathfrak{G}$, it is easy to see that $\phi: K^{t}[\mathfrak{G}] \rightarrow K^{t}[\mathbb{D}]$ is a $K^{t}[\mathbb{D}]$-bimodule homomorphism and that $\phi^{t}: K^{t}[\mathfrak{G}] \rightarrow K^{t}\left[\mathbb{D}^{t}\right]$ is a $K^{t}\left[\mathbb{D}^{t}\right]$-bimodule 
map. When $\mathfrak{G}=G$, we write $\theta, \theta^{t}, \theta_{n}$, and $\theta_{n}^{t}$ for $\phi, \phi^{t}, \phi_{n}$, and $\phi_{n}^{t}$, respectively. Additional notation will be introduced in the course of the proof.

\section{LineAR IDENTITIES}

Let $G$ be an arbitrary group and let $T$ be a subset of $G$. The (right) index $|G: T|$ is defined to be the smallest positive integer $n$ such that

$$
G=T x_{1} \cup T x_{2} \cup \cdots \cup T x_{n}
$$

for suitable $x_{1}, x_{2}, \ldots, x_{n} \in G$. Of course, if no such elements exist, then we set $|G: T|=\infty$. Note that this definition agrees with the usual index when $T$ is a subgroup of $G$.

Lemma 2.1. Let $S$ and $T$ be subsets of the group $G$.

(i) If $x \in G$, then $|G: x T|=|G: T|$.

(ii) Suppose $S=T x_{1} \cup T x_{2} \cup \cdots \cup T x_{r}$ with $x_{1}, x_{2}, \ldots, x_{r} \in G$. If $|G: S| \leq s$, then $|G: T| \leq r s$.

(iii) Assume that $S=H_{1} x_{1} \cup H_{2} x_{2} \cup \cdots \cup H_{r} x_{r}$ where each $H_{i} x_{i}$ is a coset of some subgroup $H_{i}$ of $G$. If $G=S \cup T$ and $S \neq G$, then $|G: T| \leq(r+1)$ !.

(iv) If $G=H_{1} x_{1} \cup H_{2} x_{2} \cup \cdots \cup H_{r} x_{r}$ where each $H_{i} x_{i}$ is a coset of some subgroup $H_{i}$ of $G$, then $\left|G: H_{j}\right| \leq r$ for some subscript $j$.

Proof. Parts (i) and (ii) are obvious. Parts (iii) and (iv) follow from $[8$, Lemmas 5.2.1 and 5.2.2].

Next, let $\operatorname{Hom}\left(G, K^{\bullet}\right)$ denote the dual group of $G$, namely the multiplicative group of all homomorphisms $\lambda: G \rightarrow K^{\bullet}$, where $K^{\bullet} \backslash\{0\}$. The following result generalizes Dedekind's lemma on the linear independence of characters.

Lemma 2.2. Let $K$ be a field and let $\lambda_{1}, \lambda_{2}, \ldots, \lambda_{n} \in \operatorname{Hom}\left(G, K^{\bullet}\right)$ with $\lambda_{i} \neq 1$ for all $i$. Suppose that $d_{0}, d_{1}, d_{2}, \ldots, d_{n} \in K$, and let $T$ be a subset of $G$ such that

$$
d_{0}=d_{1} \lambda_{1}(g)+d_{2} \lambda_{2}(g)+\cdots+d_{n} \lambda_{n}(g) \quad \text { for all } g \in G \backslash T \text {. }
$$

Then either $d_{0}=0$ or $|G: T| \leq 2^{n}$.

Proof. We show, by induction on $n$, that if $d_{0} \neq 0$, then $|G: T| \leq 2^{n}$. The case $n=0$ is clear, so assume that $n \geq 1$. Since $\lambda_{n} \neq 1$, we can choose $x \in G$ with $\lambda_{n}(x) \neq 1$. Then

$$
d_{0} \lambda_{n}(x)=\sum_{1}^{n} d_{i} \lambda_{i}(g) \lambda_{n}(x) \quad \text { for all } g \in G \backslash T
$$

and

$$
d_{0}=\sum_{1}^{n} d_{i} \lambda_{i}(g x)=\sum_{1}^{n} d_{i} \lambda_{i}(g) \lambda_{i}(x) \quad \text { for all } g \in G \backslash T x^{-1} .
$$

Thus, by subtracting, we obtain

$$
d_{0}\left(\lambda_{n}(x)-1\right)=\sum_{1}^{n-1} d_{i} \lambda_{i}(g)\left(\lambda_{n}(x)-\lambda_{i}(x)\right) \quad \text { for all } g \in G \backslash\left(T \cup T x^{-1}\right) .
$$

Note that $d_{0}\left(\lambda_{n}(x)-1\right) \neq 0$ so, by induction, $T \cup T x^{-1}$ has index $\leq 2^{n-1}$ in $G$. Hence $|G: T| \leq 2^{n}$ by Lemma 2.1(ii). 
Now let $K^{t}[\mathfrak{G}]$ be a twisted group algebra of $\mathfrak{G}$ over $K$, and let $G$ be a subgroup of $\mathfrak{G}$. In the following five lemmas, we suppose that $\alpha_{1}, \alpha_{2}, \ldots, \alpha_{n} \in K^{t}[\mathfrak{G}]$ and $\beta_{1}, \beta_{2}, \ldots, \beta_{n} \in K^{t}[\mathfrak{G}]$ with $A=\bigcup_{i} \operatorname{supp} \alpha_{i}, B=\bigcup_{i} \operatorname{supp} \beta_{i},|A| \leq a$ and $|B| \leq b$. For convenience, we also assume that $a \geq 2$. Recall that, if $\alpha \in K^{t}[\mathfrak{G}]$ and $x \in G$, then $\alpha^{\bar{x}}=\bar{x}^{-1} \alpha \bar{x}$. The following is the usual coset counting argument associated with the Delta Method.

Lemma 2.3. If $g \in \mathfrak{G}$, then there exists a subset $C$ of $G$ which is the set-theoretic union of at most ab right cosets of $G$-centralizers of elements of $A$, such that

$$
g \notin \operatorname{supp}\left(\alpha_{1}^{\bar{x}} \beta_{1}+\alpha_{2}^{\bar{x}} \beta_{2}+\cdots+\alpha_{n}^{\bar{x}} \beta_{n}\right) \quad \text { for all } x \in G \backslash C .
$$

Proof. Let $u \in A$ and $v \in B$. If $u$ is $G$-conjugate to $g v^{-1}$, choose $x_{u, v} \in G$ with $x_{u, v}^{-1} u x_{u, v}=g v^{-1}$. We define $C$ to be the union of the cosets $\mathbb{C}_{G}(u) x_{u, v}$ for all appropriate $u$ and $v$. Now suppose $x \in G$ and $g \in \operatorname{supp}\left(\alpha_{1}^{\bar{x}} \beta_{1}+\alpha_{2}^{\bar{x}} \beta_{2}+\cdots+\alpha_{n}^{\bar{x}} \beta_{n}\right)$. Then there must exist some $u \in A$ and $v \in B$ with $g=u^{x} v$. Thus $u^{x}=g v^{-1}$, so $x_{u, v}$ is defined, and $x^{-1} u x=g v^{-1}=x_{u, v}^{-1} u x_{u, v}$. It follows that $x x_{u, v}^{-1} \in \mathbb{C}_{G}(u)$, so $x \in \mathbb{C}_{G}(u) x_{u, v} \subseteq C$, as required.

We continue with the preceding notation. In addition, for the next four lemmas, we assume that $T$ is a subset of $G$ with

$$
\alpha_{1} \bar{x} \beta_{1}+\alpha_{2} \bar{x} \beta_{2}+\cdots+\alpha_{n} \bar{x} \beta_{n}=0 \quad \text { for all } x \in G \backslash T .
$$

For convenience, we define the integer valued function $f$, depending upon $a$ and $b$, by $f(k)=a b 2^{a-1} k^{a}$ for all integers $k \geq 1$. It is clear that $f(k) \geq k$ provided $a, b \geq 1$. At this point, the notation of the preceding section comes into play.

Lemma 2.4. Let $k \geq 1$ be an integer and assume that $A \cap \mathbb{D}_{f(k)}=A \cap \mathbb{D}_{k}$ and that $A \cap \mathbb{D}_{f(k)}^{t}=A \cap \mathbb{D}_{k}^{t}$. Then either $|G: T| \leq k^{a}\left(a b 2^{a-1}+2\right)$ ! or

$$
\phi_{k}^{t}\left(\alpha_{1}\right) \beta_{1}+\phi_{k}^{t}\left(\alpha_{2}\right) \beta_{2}+\cdots+\phi_{k}^{t}\left(\alpha_{n}\right) \beta_{n}=0 .
$$

Proof. We will show that if $\phi_{k}^{t}\left(\alpha_{1}\right) \beta_{1}+\phi_{k}^{t}\left(\alpha_{2}\right) \beta_{2}+\cdots+\phi_{k}^{t}\left(\alpha_{n}\right) \beta_{n} \neq 0$, then $|G: T|$ is suitably bounded. To this end, write $A=R \cup S \cup Z$ where $R=A \cap \mathbb{D}_{k}^{t}$, $S=\left(A \cap \mathbb{D}_{k}\right) \backslash R$ and $Z=A \backslash(R \cup S)$. Then $R, S$ and $Z$ are disjoint and we can write each $\alpha_{i}$ uniquely as $\alpha_{i}=\rho_{i}-\sigma_{i}+\zeta_{i}$ where $\operatorname{supp} \rho_{i} \subseteq R$, supp $\sigma_{i} \subseteq S$ and $\operatorname{supp} \zeta_{i} \subseteq Z$. It is clear that $\rho_{i}=\phi_{k}^{t}\left(\alpha_{i}\right)$. In particular, $|R| \geq 1$.

Set

$$
W=\bigcap_{u \in R} \mathbb{C}_{G}^{t}(u) \cap \bigcap_{u \in S} \mathbb{C}_{G}(u) .
$$

Then, by definition, each of the intersecting subgroups here has index at most $k$, so $|R \cup S| \leq|A|=a$ implies that $|G: W| \leq k^{a}$. Now let $g \in \mathfrak{G}$ be a fixed element in the support of $\phi_{k}^{t}\left(\alpha_{1}\right) \beta_{1}+\phi_{k}^{t}\left(\alpha_{2}\right) \beta_{2}+\cdots+\phi_{k}^{t}\left(\alpha_{n}\right) \beta_{n} \neq 0$, and let $0 \neq d \in K$ be the coefficient of $\bar{g}$. For convenience, set $T^{\prime}=T \cap W$. We will bound $\left|G: T^{\prime}\right| \geq|G: T|$.

Let $x \in W \backslash T^{\prime}$. Then $x \in G \backslash T$, so

$$
0=\bar{x}^{-1} \sum_{i} \alpha_{i} \bar{x} \beta_{i}=\sum_{i} \alpha_{i}^{\bar{x}} \beta_{i}=\sum_{i} \rho_{i}^{\bar{x}} \beta_{i}-\sum_{i} \sigma_{i}^{\bar{x}} \beta_{i}+\sum_{i} \zeta_{i}^{\bar{x}} \beta_{i}
$$

Note that $W \subseteq \mathbb{C}_{G}^{t}(R)$, by definition of $W$, so $\rho_{i}^{\bar{x}}=\rho_{i}$. Furthermore, Lemma 2.3 implies that there exists a subset $C$ of $W$, that is a union of at most $a b$ right cosets of $W$-centralizers of elements of $Z$, with

$$
g \notin \operatorname{supp} \sum_{i} \zeta_{i}^{\bar{x}} \beta_{i} \quad \text { for all } x \in W \backslash C .
$$


Thus, for all $x \in W \backslash\left(T^{\prime} \cup C\right)$, the coefficient of $\bar{g}$ in

$$
\sum_{i} \sigma_{i}^{\bar{x}} \beta_{i}=\sum_{i} \rho_{i} \beta_{i}+\sum_{i} \zeta_{i}^{\bar{x}} \beta_{i}
$$

is precisely equal to $d \neq 0$.

Now if $u \in \operatorname{supp} \sigma_{i} \subseteq S$, then $W$ centralizes $u$, by the definition of $W$. In particular, for all $x \in W, \bar{u}^{\bar{x}}=\lambda_{u}(x) \bar{u}$ where $\lambda_{u}: W \rightarrow K^{\bullet}$ is a linear character. It follows that the coefficient of $\bar{g}$ in $\sum_{i} \sigma_{i}^{\bar{x}} \beta_{i}$ is a fixed $K$-linear combination of the various $\lambda_{u}(x)$. In other words, there exist $d_{u} \in K$ with

$$
0 \neq d=\sum_{u \in S} d_{u} \lambda_{u}(x) \quad \text { for all } x \in W \backslash\left(T^{\prime} \cup C\right) .
$$

We note that $\lambda_{u} \neq 1$ for all $u \in S$. Indeed, if $\lambda_{u}=1$, then $\mathbb{C}_{G}^{t}(u) \supseteq W$. Hence $\left|G: \mathbb{C}_{G}^{t}(u)\right| \leq|G: W| \leq k^{a} \leq f(k)$ and $u \in A \cap \mathbb{D}_{f(k)}^{t}=A \cap \mathbb{D}_{k}^{t}=R$, a contradiction. Thus, since $|S| \leq|A|-|R| \leq a-1$, we can apply Lemma 2.2 to conclude that $\left|W: T^{\prime} \cup C\right| \leq 2^{a-1}$.

Let $Y$ be a subset of $W$ of size $\leq 2^{a-1}$ with $W=\left(T^{\prime} \cup C\right) Y=T^{\prime} Y \cup C Y$. We claim that $C Y \neq W$. Indeed, since $C$ is a union of $\leq a b$ cosets of $W$-centralizers of elements of $Z$, the equality $C Y=W$ along with Lemma 2.1(iv) would imply that $\left|W: \mathbb{C}_{W}(u)\right| \leq a b 2^{a-1}$ for some $u \in Z$. But then

$$
\left|G: \mathbb{C}_{G}(u)\right| \leq|G: W|\left|W: \mathbb{C}_{W}(u)\right| \leq a b 2^{a-1} k^{a}=f(k),
$$

so $u \in A \cap \mathbb{D}_{f(k)}=A \cap \mathbb{D}_{k}$, by hypothesis. In particular, $u \in R \cup S$, a contradiction. Thus, $C Y \neq W$, so $W=T^{\prime} Y \cup C Y$ and Lemma 2.1(iii) yield the inequality $\left|W: T^{\prime} Y\right| \leq\left(a b 2^{a-1}+1\right)$ ! and hence $\left|W: T^{\prime}\right| \leq 2^{a-1}\left(a b 2^{a-1}+1\right) ! \leq\left(a b 2^{a-1}+2\right)$ ! since $|Y| \leq 2^{a-1}$. Furthermore, by Lemma 2.1(ii) again,

$$
|G: T| \leq\left|G: T^{\prime}\right| \leq|G: W|\left|W: T^{\prime}\right| \leq k^{a}\left(a b 2^{a-1}+2\right) !
$$

and $|G: T|$ is suitably bounded.

As an immediate consequence, we obtain

Lemma 2.5. Let $k \geq 1$ be an integer and assume that $A \cap \mathbb{D}_{f(k)}=A \cap \mathbb{D}_{k}$ and $A \cap \mathbb{D}_{f(k)}^{t}=A \cap \mathbb{D}_{k}^{t}$. Then either $|G: T| \leq k^{a}\left(a b 2^{a-1}+2\right)$ ! or

$$
\phi_{k}^{t}\left(\alpha_{1}\right) \bar{x} \beta_{1}+\phi_{k}^{t}\left(\alpha_{2}\right) \bar{x} \beta_{2}+\cdots+\phi_{k}^{t}\left(\alpha_{n}\right) \bar{x} \beta_{n}=0 \quad \text { for all } x \in G .
$$

Proof. We suppose that $|G: T|>k^{a}\left(a b 2^{a-1}+2\right)$ !. Let $g \in G$ be arbitrary and note that

$$
\alpha_{1} \bar{x} \bar{g} \beta_{1}+\alpha_{2} \bar{x} \bar{g} \beta_{2}+\cdots+\alpha_{n} \bar{x} \bar{g} \beta_{n}=0 \quad \text { for all } x \in G \backslash T g^{-1},
$$

since $\bar{x} \bar{g}=d \overline{x g}$ for some $0 \neq d \in K$. Furthermore, if $B^{\prime}=\bigcup_{i} \operatorname{supp} \bar{g} \beta_{i}$, then $B^{\prime}=g B$, so $\left|B^{\prime}\right|=|B|=b$. Thus, since $\left|G: T g^{-1}\right|=|G: T|$ is suitably large, Lemma 2.4 implies that

$$
\phi_{k}^{t}\left(\alpha_{1}\right) \bar{g} \beta_{1}+\phi_{k}^{t}\left(\alpha_{2}\right) \bar{g} \beta_{2}+\cdots+\phi_{k}^{t}\left(\alpha_{n}\right) \bar{g} \beta_{n}=0,
$$

and the result follows since this holds for all $g \in G$.

Now let $r=2 \max \{a, b\}$ and note that if $k \geq 4$ then $f(k) \leq k^{r}$. Indeed, if $m=\max \{a, b\}$, then $2^{m+1} \geq m^{2}$, so $k \geq 4$ yields $k^{m} \geq 2^{2 m} \geq m^{2} 2^{m-1} \geq a b 2^{a-1}$ and hence $k^{r} \geq k^{m+a} \geq a b 2^{a-1} k^{a}=f(k)$. In the next lemma, we use a pigeon-hole argument to show that 
Lemma 2.6. Either $|G: T| \leq(1 / r)^{3} \cdot 4^{r^{r+1}}$ ! or there exists an integer $k \leq 4^{r^{r}}$ with

$$
\phi_{k}^{t}\left(\alpha_{1}\right) \bar{x} \beta_{1}+\phi_{k}^{t}\left(\alpha_{2}\right) \bar{x} \beta_{2}+\cdots+\phi_{k}^{t}\left(\alpha_{n}\right) \bar{x} \beta_{n}=0 \quad \text { for all } x \in G \text {. }
$$

Proof. Let $s_{i}=4^{r^{i}}$ for $i \geq 0$ and note that $4=s_{0} \leq s_{1} \leq s_{2} \leq \cdots$. Hence the subsets $A \cap\left(\mathbb{D}_{s_{i+1}}^{t} \backslash \mathbb{D}_{s_{i}}^{t}\right)$ are disjoint, and consequently at most $a=|A|$ of these can be nonempty. Similarly, at most $a$ of the subsets $A \cap\left(\mathbb{D}_{s_{i+1}} \backslash \mathbb{D}_{s_{i}}\right)$ can be nonempty. Since $r \geq 2 a$, it follows that there exists a subscript $i$ with $0 \leq i \leq r$ and with $A \cap\left(\mathbb{D}_{s_{i+1}}^{t} \backslash \mathbb{D}_{s_{i}}^{t}\right)=\emptyset=A \cap\left(\mathbb{D}_{s_{i+1}} \backslash \mathbb{D}_{s_{i}}\right)$. If $k=s_{i}$, then $4 \leq k \leq s_{r}=\overline{4}^{r^{r}}$ and, as we observed, $f(k) \leq k^{r}=s_{i}^{r}=s_{i+1}$. Thus $A \cap \mathbb{D}_{f(k)}^{t}=A \cap \mathbb{D}_{k}^{t}$ and $A \cap \mathbb{D}_{f(k)}=A \cap \mathbb{D}_{k}$, so Lemma 2.5 implies that either

$$
\phi_{k}^{t}\left(\alpha_{1}\right) \bar{x} \beta_{1}+\phi_{k}^{t}\left(\alpha_{2}\right) \bar{x} \beta_{2}+\cdots+\phi_{k}^{t}\left(\alpha_{n}\right) \bar{x} \beta_{n}=0 \quad \text { for all } x \in G
$$

or

$$
\begin{aligned}
|G: T| & \leq k^{a}\left(a b 2^{a-1}+2\right) ! \leq(1 / 8 a b)^{3} \cdot\left(a b 2^{a-1} k^{a}\right) ! \\
& \leq(1 / r)^{3} \cdot f(k) ! \leq(1 / r)^{3} \cdot s_{r+1} !=(1 / r)^{3} \cdot 4^{r^{r+1}} !
\end{aligned}
$$

since $k^{a} \geq 4^{2}=16$, and the lemma is proved.

The next result is actually a special case of the more important Proposition 2.8. It is certainly simpler to state.

Lemma 2.7. Either $|G: T| \leq 4^{r^{r+1}}$ ! or

$$
\phi^{t}\left(\alpha_{1}\right) \bar{x} \beta_{1}+\phi^{t}\left(\alpha_{2}\right) \bar{x} \beta_{2}+\cdots+\phi^{t}\left(\alpha_{n}\right) \bar{x} \beta_{n}=0 \quad \text { for all } x \in G .
$$

Proof. Assume that $|G: T|>4^{r^{r+1}}$ !. We proceed by induction on $\left|A \cap \mathbb{D}^{t}\right|$ to show that $\phi^{t}\left(\alpha_{1}\right) \bar{x} \beta_{1}+\phi^{t}\left(\alpha_{2}\right) \bar{x} \beta_{2}+\cdots+\phi^{t}\left(\alpha_{n}\right) \bar{x} \beta_{n}=0$ for all $x \in G$. The result is, of course, clear if $A \cap \mathbb{D}^{t}=\emptyset$. So suppose $g \in A \cap \mathbb{D}^{t}$ and observe that

$$
\alpha_{1}^{\prime} \bar{x} \beta_{1}+\alpha_{2}^{\prime} \bar{x} \beta_{2}+\cdots+\alpha_{n}^{\prime} \bar{x} \beta_{n}=0 \quad \text { for all } x \in G \backslash T,
$$

where $\alpha_{i}^{\prime}=\bar{g}^{-1} \alpha_{i}$. Furthermore, if $A^{\prime}=\bigcup_{i} \operatorname{supp} \alpha_{i}^{\prime}$, then $A^{\prime}=g^{-1} A$ and $A^{\prime} \cap \mathbb{D}^{t}=$ $g^{-1}\left(A \cap \mathbb{D}^{t}\right)$, since $g \in \mathbb{D}^{t}$. Thus the corresponding sets have the same size, and $1 \in A^{\prime} \cap \mathbb{D}^{t}$. By the preceding lemma and the assumption on $|G: T|$, there exists an integer $k$ with

$$
\phi_{k}^{t}\left(\alpha_{1}^{\prime}\right) \bar{x} \beta_{1}+\phi_{k}^{t}\left(\alpha_{2}^{\prime}\right) \bar{x} \beta_{2}+\cdots+\phi_{k}^{t}\left(\alpha_{n}^{\prime}\right) \bar{x} \beta_{n}=0 \quad \text { for all } x \in G .
$$

Hence, setting $\alpha_{i}^{\prime \prime}=\alpha_{i}^{\prime}-\phi_{k}^{t}\left(\alpha_{i}^{\prime}\right)$, we have

$$
\alpha_{1}^{\prime \prime} \bar{x} \beta_{1}+\alpha_{2}^{\prime \prime} \bar{x} \beta_{2}+\cdots+\alpha_{n}^{\prime \prime} \bar{x} \beta_{n}=0 \quad \text { for all } x \in G \backslash T \text {. }
$$

Note that $A^{\prime \prime}=\bigcup_{i} \operatorname{supp} \alpha_{i}^{\prime \prime} \subseteq \bigcup_{i} \operatorname{supp} \alpha_{i}^{\prime}=A^{\prime}$ and that $1 \in A^{\prime} \cap \mathbb{D}^{t}$ but $1 \notin$ $A^{\prime \prime} \cap \mathbb{D}^{t}$ since $1 \in A^{\prime} \cap \mathbb{D}_{k}^{t}$. Thus $\left|A^{\prime \prime} \cap \mathbb{D}^{t}\right|<\left|A^{\prime} \cap \mathbb{D}^{t}\right|$ and, by induction,

$$
\phi^{t}\left(\alpha_{1}^{\prime \prime}\right) \bar{x} \beta_{1}+\phi^{t}\left(\alpha_{2}^{\prime \prime}\right) \bar{x} \beta_{2}+\cdots+\phi^{t}\left(\alpha_{n}^{\prime \prime}\right) \bar{x} \beta_{n}=0 \quad \text { for all } x \in G .
$$

Finally, note that $\phi^{t}\left(\alpha_{i}^{\prime \prime}\right)=\phi^{t}\left(\alpha_{i}^{\prime}-\phi_{k}^{t}\left(\alpha_{i}^{\prime}\right)\right)=\phi^{t}\left(\alpha_{i}^{\prime}\right)-\phi_{k}^{t}\left(\alpha_{i}^{\prime}\right)$, so

$$
\begin{aligned}
\phi^{t}\left(\alpha_{1}^{\prime}\right) \bar{x} \beta_{1} & +\phi^{t}\left(\alpha_{2}^{\prime}\right) \bar{x} \beta_{2}+\cdots+\phi^{t}\left(\alpha_{n}^{\prime}\right) \bar{x} \beta_{n} \\
& =\phi_{k}^{t}\left(\alpha_{1}^{\prime}\right) \bar{x} \beta_{1}+\phi_{k}^{t}\left(\alpha_{2}^{\prime}\right) \bar{x} \beta_{2}+\cdots+\phi_{k}^{t}\left(\alpha_{n}^{\prime}\right) \bar{x} \beta_{n}=0 \quad \text { for all } x \in G .
\end{aligned}
$$

Thus, since $\phi^{t}\left(\alpha_{i}^{\prime}\right)=\phi^{t}\left(\bar{g}^{-1} \alpha_{i}\right)=\bar{g}^{-1} \phi^{t}\left(\alpha_{i}\right)$, we conclude that

$$
\phi^{t}\left(\alpha_{1}\right) \bar{x} \beta_{1}+\phi^{t}\left(\alpha_{2}\right) \bar{x} \beta_{2}+\cdots+\phi^{t}\left(\alpha_{n}\right) \bar{x} \beta_{n}=0 \quad \text { for all } x \in G,
$$

as required. 
The following is the key result needed to handle generalized polynomial identities. We state it in a somewhat cleaner form using singletons, that is elements of the twisted group ring of support size $\leq 1$. Alternately, we could state the result in terms of the various truncations of the elements $\gamma_{j}$. Recall that a truncation of an element in a twisted group ring is a partial sum of the natural expression of that element as a $K$-linear combination of members of the basis $\overline{\mathfrak{G}}$. Thus, for example, $\phi^{t}(\alpha)$ and $\alpha-\phi^{t}(\alpha)$ are both truncations of $\alpha \in K^{t}[\mathfrak{G}]$. Note also that any truncation of a singleton is either itself or 0 .

Proposition 2.8. Let $K^{t}[\mathfrak{G}]$ be a twisted group algebra and let $\alpha_{i}, \beta_{i}, \gamma_{j}, \delta_{j} \in K^{t}[\mathfrak{G}]$ with $i=1,2, \ldots, n$ and $j=1,2, \ldots, m$. Assume that each $\gamma_{j}$ is a singleton. Let $G$ be a subgroup of $\mathfrak{G}$, let $T$ be a subset of $G$, and suppose that

$$
\sum_{i=1}^{n} \alpha_{i} \bar{x} \beta_{i}+\sum_{j=1}^{m} \gamma_{j} \bar{x} \delta_{j}=0 \quad \text { for all } x \in G \backslash T .
$$

Write $A=\bigcup_{i} \operatorname{supp} \alpha_{i}, B=\bigcup_{i} \operatorname{supp} \beta_{i}, C=\bigcup_{j} \operatorname{supp} \gamma_{j}, D=\bigcup_{j} \operatorname{supp} \delta_{j}$, and let $r \geq 2 \max \{|A \cup C|,|B \cup D|\}$ with $r \geq 4$. Then either $|G: T| \leq(1 / r)^{3} \cdot 4^{r^{r+1}}$ ! or

$$
\sum_{i=1}^{n} \phi^{t}\left(\alpha_{i}\right) \bar{x} \beta_{i}+\sum_{j=1}^{m} \psi\left(\gamma_{j}\right) \bar{x} \delta_{j}=0 \quad \text { for all } x \in G
$$

where $\psi\left(\gamma_{j}\right)$ is equal to 0 or to $\bar{y} \phi_{k}^{t}\left(\bar{y}^{-1} \gamma_{j}\right)$ for some $y \in A \cap \mathbb{D}^{t}$ and some integer $k \leq 4^{r^{r}}$. In particular, if $\gamma_{j}=0$, then $\psi\left(\gamma_{j}\right)=0$.

Proof. Assume that $|G: T|>(1 / r)^{3} \cdot 4^{r^{r+1}}$ !. We proceed by induction on $\left|A \cap \mathbb{D}^{t}\right|$ to show that $\sum_{i} \phi^{t}\left(\alpha_{i}\right) \bar{x} \beta_{i}+\sum_{j} \psi\left(\gamma_{j}\right) \bar{x} \delta_{j}=0$ for all $x \in G$ and for appropriate $\psi\left(\gamma_{j}\right)$. The result is, of course, clear if $A \cap \mathbb{D}^{t}=\emptyset$. So suppose $g \in A \cap \mathbb{D}^{t}$ and observe that

$$
\sum_{i=1}^{n} \alpha_{i}^{\prime} \bar{x} \beta_{i}+\sum_{j=1}^{m} \gamma_{j}^{\prime} \bar{x} \delta_{j}=0 \quad \text { for all } x \in G \backslash T,
$$

where $\alpha_{i}^{\prime}=\bar{g}^{-1} \alpha_{i}$ and $\gamma_{j}^{\prime}=\bar{g}^{-1} \gamma_{j}$. Furthermore, if $A^{\prime}=\bigcup_{i} \operatorname{supp} \alpha_{i}^{\prime}$ and $C^{\prime}=$ $\bigcup_{j} \operatorname{supp} \gamma_{j}^{\prime}$, then $A^{\prime}=g^{-1} A, C^{\prime}=g^{-1} C$ and $A^{\prime} \cap \mathbb{D}^{t}=g^{-1}\left(A \cap \mathbb{D}^{t}\right)$, since $g \in \mathbb{D}^{t}$. Thus the corresponding sets have the same size, and $1 \in A^{\prime} \cap \mathbb{D}^{t}$. By Lemma 2.6 and the assumption on $|G: T|$, there exists an integer $k \leq 4^{r^{r}}$ with

$$
\sum_{i=1}^{n} \phi_{k}^{t}\left(\alpha_{i}^{\prime}\right) \bar{x} \beta_{i}+\sum_{j=1}^{m} \phi_{k}^{t}\left(\gamma_{j}^{\prime}\right) \bar{x} \delta_{j}=0 \quad \text { for all } x \in G .
$$

Hence, setting $\alpha_{i}^{\prime \prime}=\alpha_{i}^{\prime}-\phi_{k}^{t}\left(\alpha_{i}^{\prime}\right)$ and $\gamma_{j}^{\prime \prime}=\gamma_{j}^{\prime}-\phi_{k}^{t}\left(\gamma_{j}^{\prime}\right)$, we have

$$
\sum_{i=1}^{n} \alpha_{i}^{\prime \prime} \bar{x} \beta_{i}+\sum_{j=1}^{m} \gamma_{j}^{\prime \prime} \bar{x} \delta_{j}=0 \quad \text { for all } x \in G \backslash T .
$$

Note that $A^{\prime \prime}=\bigcup_{i} \operatorname{supp} \alpha_{i}^{\prime \prime} \subseteq \bigcup_{i} \operatorname{supp} \alpha_{i}^{\prime}=A^{\prime}$ and that $1 \in A^{\prime} \cap \mathbb{D}^{t}$ but $1 \notin$ $A^{\prime \prime} \cap \mathbb{D}^{t}$ since $1 \in A^{\prime} \cap \mathbb{D}_{k}^{t}$. Furthermore, $C^{\prime \prime}=\bigcup_{j} \operatorname{supp} \gamma_{j}^{\prime \prime} \subseteq \bigcup_{j} \operatorname{supp} \gamma_{j}^{\prime}=C^{\prime}$, and $\gamma_{j}^{\prime \prime}=\gamma_{j}^{\prime}-\phi_{k}^{t}\left(\gamma_{j}^{\prime}\right)$ is a truncation of $\gamma_{j}^{\prime}$ and hence it is also a singleton. Thus, since $\left|A^{\prime \prime} \cap \mathbb{D}^{t}\right|<\left|A^{\prime} \cap \mathbb{D}^{t}\right|$, induction implies that

$$
\sum_{i=1}^{n} \phi^{t}\left(\alpha_{i}^{\prime \prime}\right) \bar{x} \beta_{i}+\sum_{j=1}^{m} \psi\left(\gamma_{j}^{\prime \prime}\right) \bar{x} \delta_{j}=0 \quad \text { for all } x \in G
$$


and for appropriate $\psi\left(\gamma_{j}^{\prime \prime}\right)$. Next, $\phi^{t}\left(\alpha_{i}^{\prime \prime}\right)=\phi^{t}\left(\alpha_{i}^{\prime}-\phi_{k}^{t}\left(\alpha_{i}^{\prime}\right)\right)=\phi^{t}\left(\alpha_{i}^{\prime}\right)-\phi_{k}^{t}\left(\alpha_{i}^{\prime}\right)$, so $\phi^{t}\left(\alpha_{i}^{\prime}\right)=\phi^{t}\left(\alpha_{i}^{\prime \prime}\right)+\phi_{k}^{t}\left(\alpha_{i}^{\prime}\right)$ and, by adding $(*)$ and $(* *)$, we obtain

$$
\sum_{i=1}^{n} \phi^{t}\left(\alpha_{i}^{\prime}\right) \bar{x} \beta_{i}+\sum_{j=1}^{m}\left[\phi_{k}^{t}\left(\gamma_{j}^{\prime}\right)+\psi\left(\gamma_{j}^{\prime \prime}\right)\right] \bar{x} \delta_{j}=0 \quad \text { for all } x \in G .
$$

Thus, since $\phi^{t}\left(\alpha_{i}^{\prime}\right)=\phi^{t}\left(\bar{g}^{-1} \alpha_{i}\right)=\bar{g}^{-1} \phi^{t}\left(\alpha_{i}\right)$, we conclude that

$$
\sum_{i=1}^{n} \phi^{t}\left(\alpha_{i}\right) \bar{x} \beta_{i}+\sum_{j=1}^{m} \psi\left(\gamma_{j}\right) \bar{x} \delta_{j}=0 \quad \text { for all } x \in G,
$$

where we set $\psi\left(\gamma_{j}\right)=\bar{g} \phi_{k}^{t}\left(\gamma_{j}^{\prime}\right)+\bar{g} \psi\left(\gamma_{j}^{\prime \prime}\right)=\bar{g} \phi_{k}^{t}\left(\bar{g}^{-1} \gamma_{j}\right)+\bar{g} \psi\left(\gamma_{j}^{\prime \prime}\right)$. It remains to better understand $\psi\left(\gamma_{j}\right)$.

To this end, suppose first that $\bar{g} \phi_{k}^{t}\left(\gamma_{j}^{\prime}\right)=\bar{g} \phi_{k}^{t}\left(\bar{g}^{-1} \gamma_{j}\right) \neq 0$. Then since $\gamma_{j}^{\prime}=$ $\bar{g}^{-1} \gamma_{j}$ is a singleton, it follows that $\gamma_{j}^{\prime \prime}=\gamma_{j}^{\prime}-\phi_{k}^{t}\left(\gamma_{j}^{\prime}\right)=0$. Hence $\psi\left(\gamma_{j}^{\prime \prime}\right)=0$ and $\psi\left(\gamma_{j}\right)=\bar{g} \phi_{k}^{t}\left(\bar{g}^{-1} \gamma_{j}\right)$, as required. On the other hand, if $\bar{g} \phi_{k}^{t}\left(\gamma_{j}^{\prime}\right)=\bar{g} \phi_{k}^{t}\left(\bar{g}^{-1} \gamma_{j}\right)=0$, then $\gamma_{j}^{\prime \prime}=\gamma_{j}^{\prime}=\bar{g}^{-1} \gamma_{j}$ is a singleton and $\psi\left(\gamma_{j}\right)=\bar{g} \psi\left(\gamma_{j}^{\prime \prime}\right)$ is either 0 or equal to $\bar{g} \bar{y} \theta_{\ell}^{t}\left(\bar{y}^{-1} \gamma_{j}^{\prime \prime}\right)=\bar{g} \bar{y} \theta_{\ell}^{t}\left(\bar{y}^{-1} \bar{g}^{-1} \gamma_{j}\right)$ for some $y \in A^{\prime \prime} \cap \mathbb{D}^{t}$ and $\ell \leq 4^{r^{r}}$. Note that, in the latter case, $g y \in \mathbb{D}^{t}$ and $y \in A^{\prime \prime} \subseteq A^{\prime}=g^{-1} A$ so $g y \in A$. Thus $g y \in A \cap \mathbb{D}^{t}$ and, since $\overline{g y}=d \bar{g} \bar{y}$ for some $0 \neq d \in K$, we conclude that $\psi\left(\gamma_{j}\right)=\overline{g y} \theta_{\ell}^{t}\left(\overline{g y}^{-1} \gamma_{j}\right)$ also has the appropriate form.

\section{Generalized Polynomial Identities}

We now move on to consider multilinear generalized polynomial identities. Since these identities are, in some sense, combinations of linear identities, it is not surprising that the work of the previous section should come into play. Fortunately, the arguments here are somewhat less computational.

In this section, we assume that $K^{t}[\mathfrak{G}]$ is given and that $G$ is a normal subgroup of $\mathfrak{G}$. As we observed, the latter implies that $\mathbb{D}, \mathbb{D}^{t}, \mathbb{D}_{k}$ and $\mathbb{D}_{k}^{t}$ are all normal subsets of $\mathfrak{G}$. Let

$$
f\left(\zeta_{1}, \zeta_{2}, \ldots, \zeta_{n}\right)=\sum_{\sigma \in \operatorname{Sym}_{n}} \sum_{j=1}^{a_{\sigma}} \alpha_{0, \sigma, j} \zeta_{\sigma(1)} \alpha_{1, \sigma, j} \zeta_{\sigma(2)} \cdots \alpha_{n-1, \sigma, j} \zeta_{\sigma(n)} \alpha_{n, \sigma, j}
$$

be a multilinear generalized polynomial with each $\alpha_{i, \sigma, j}$ in $K^{t}[\mathfrak{G}]$, and assume throughout that $K^{t}[G]$ satisfies $f$, so that

$$
f\left(\gamma_{1}, \gamma_{2}, \ldots, \gamma_{n}\right)=0 \quad \text { for all } \gamma_{1}, \gamma_{2}, \ldots, \gamma_{n} \in K^{t}[G] .
$$

As usual, for each $\sigma \in \mathrm{Sym}_{n}$, we write

$$
f_{\sigma}\left(\zeta_{1}, \zeta_{2}, \ldots, \zeta_{n}\right)=\sum_{j=1}^{a_{\sigma}} \alpha_{0, \sigma, j} \zeta_{\sigma(1)} \alpha_{1, \sigma, j} \zeta_{\sigma(2)} \cdots \alpha_{n-1, \sigma, j} \zeta_{\sigma(n)} \alpha_{n, \sigma, j}
$$

for the $\sigma$-component of $f$. We start with

Lemma 3.1. Let $K^{t}[\mathfrak{G}], G$, and $f$ be as above. Assume further that each $\alpha_{i, \sigma, j}$ is a singleton and that

$$
\sum_{j=1}^{a_{1}} \phi^{t}\left(\alpha_{0,1, j}\right) \phi^{t}\left(\alpha_{1,1, j}\right) \cdots \phi^{t}\left(\alpha_{n, 1, j}\right) \neq 0 .
$$


If $r \geq 2 \sum_{\sigma} a_{\sigma}$, twice the number of monomials which occur in $f$, and if $k=4^{r^{r}}$, then $\left|G: \Delta_{k^{2}}^{t}\right| \leq(n / r) \cdot 4^{r^{r+1}}$ !.

Proof. For each $i=0,1, \ldots, n$, define $S_{i} \subseteq \mathrm{Sym}_{n}$ by

$$
S_{i}=\left\{\sigma \in \operatorname{Sym}_{n} \mid \sigma(1)=1, \sigma(2)=2, \ldots, \sigma(i)=i\right\} .
$$

Then $S_{0}=\operatorname{Sym}_{n}, S_{n}=\{1\}$ and $S_{i} \cong \operatorname{Sym}_{n-i}$. Using this, we define the generalized multilinear polynomial $f_{i}$ of degree $n-i$ by

$$
f_{i}\left(\zeta_{i+1}, \zeta_{i+2}, \ldots, \zeta_{n}\right)=\sum_{\sigma \in S_{i}} \sum_{j=1}^{a_{\sigma}} \beta_{i, \sigma, j} \zeta_{\sigma(i+1)} \alpha_{i+1, \sigma, j} \cdots \alpha_{n-1, \sigma, j} \zeta_{\sigma(n)} \alpha_{n, \sigma, j}
$$

where

$$
\beta_{i, \sigma, j}=\phi^{t}\left(\alpha_{0, \sigma, j}\right) \phi^{t}\left(\alpha_{1, \sigma, j}\right) \cdots \phi^{t}\left(\alpha_{i-1, \sigma, j}\right) \alpha_{i, \sigma, j} .
$$

Then $f_{0}=f$ and

$$
f_{n}=\sum_{j=1}^{a_{1}} \beta_{n, 1, j}=\sum_{j=1}^{a_{1}} \phi^{t}\left(\alpha_{0,1, j}\right) \phi^{t}\left(\alpha_{1,1, j}\right) \cdots \phi^{t}\left(\alpha_{n-1,1, j}\right) \alpha_{n, 1, j}
$$

is a nonzero element of $K^{t}[\mathfrak{G}]$ since

$$
\phi^{t}\left(f_{n}\right)=\sum_{j=1}^{a_{1}} \phi^{t}\left(\alpha_{0,1, j}\right) \phi^{t}\left(\alpha_{1,1, j}\right) \cdots \phi^{t}\left(\alpha_{n-1,1, j}\right) \phi^{t}\left(\alpha_{n, 1, j}\right) \neq 0 .
$$

Next, set $\mathcal{M}_{0}^{\prime}=\emptyset$ and, for $i=1,2, \ldots, n$, let $\mathcal{M}_{i}^{\prime}$ to be the set of all monomials $\mu$ of the form

$$
\mu=\beta_{i-1, \tau, j^{\prime}}^{-1} \beta_{i-1, \sigma, j} \zeta_{\sigma(i)} \alpha_{i, \sigma, j} \zeta_{\sigma(i+1)} \alpha_{i+1, \sigma, j} \cdots \alpha_{\ell-2, \sigma, j} \zeta_{\sigma(\ell-1)} \alpha_{\ell-1, \sigma, j}
$$

with $\sigma \in S_{i-1} \backslash S_{i}, \sigma(\ell)=i$, and $\tau \in S_{i}$. Furthermore, we must have $\beta_{i-1, \tau, j^{\prime}} \neq 0$ and, since this element is a singleton, it is invertible in $K^{t}[\mathfrak{G}]$. Note that any such $\mu$ involves only variables from the set $\left\{\zeta_{i+1}, \zeta_{i+2}, \ldots, \zeta_{n}\right\}$ and that at least one variable occurs since $\sigma \in S_{i-1} \backslash S_{i}$ implies that $\sigma(i) \neq i$. Furthermore, since $\mu$ is uniquely determined by the pairs $\{\sigma, j\}$ and $\left\{\tau, j^{\prime}\right\}$, we see that $\left|\mathcal{M}_{i}^{\prime}\right| \leq r^{2}$ and that $\mathcal{M}_{n}^{\prime}=\emptyset$. Now let $\mathcal{M}=\bigcup_{i=0}^{n} \mathcal{M}_{i}^{\prime}$ and let $\mathcal{M}_{i}$ denote the set of monomials in $\mathcal{M}$ which involve only variables in the set $\left\{\zeta_{i+1}, \zeta_{i+2}, \ldots, \zeta_{n}\right\}$. Then clearly $|\mathcal{M}| \leq n r^{2}$, $\mathcal{M}_{i} \supseteq \mathcal{M}_{i}^{\prime}$, and $\mathcal{M}=\mathcal{M}_{0} \supseteq \mathcal{M}_{1} \supseteq \cdots \supseteq \mathcal{M}_{n}=\emptyset$.

Set $k=4^{r^{r}}$. Our goal is to show that $\left|G: \Delta_{k^{2}}^{t}\right| \leq(n / r) \cdot 4^{r^{r+1}}$ !. Suppose, by way of contradiction, that this is not the case. Then we will prove by induction on $i=0,1, \ldots, n$ that, for all $x_{i+1}, x_{i+2}, \ldots, x_{n} \in G$, either $f_{i}\left(\bar{x}_{i+1}, \bar{x}_{i+2}, \ldots, \bar{x}_{n}\right)=0$ or there exists a monomial $\mu$ in $\mathcal{M}_{i}$ with $\operatorname{supp} \mu\left(\bar{x}_{i+1}, \bar{x}_{i+2}, \ldots, \bar{x}_{n}\right) \cap \mathbb{D}_{k}^{t} \neq \emptyset$. This is, of course, clear for $i=0$ since $f_{0}=f$ is a generalized polynomial identity satisfied by $K^{t}[G]$. Assume now that $1 \leq i \leq n$ and that the result holds for $i-1$. Fix the elements $x_{i+1}, x_{i+2}, \ldots, x_{n} \in G$ and let $x \in G$ play the role of the $i$ th variable. Let $\mu \in \mathcal{M}_{i}$. If $\operatorname{supp} \mu\left(\bar{x}_{i+1}, \bar{x}_{i+2}, \ldots, \bar{x}_{n}\right) \cap \mathbb{D}_{k}^{t} \neq \emptyset$, we are done. Thus we may suppose that $\operatorname{supp} \mu\left(\bar{x}_{i+1}, \bar{x}_{i+2}, \ldots, \bar{x}_{n}\right) \cap \mathbb{D}_{k}^{t}=\emptyset$ for all $\mu \in \mathcal{M}_{i}$.

Now let $\mu \in \mathcal{M}_{i-1} \backslash \mathcal{M}_{i}=\mathcal{N}_{i-1}$ so that $\mu$ involves the variable $\zeta_{i}$. Write $\mu=\mu^{\prime} \zeta_{i} \mu^{\prime \prime}$ where $\mu^{\prime}$ and $\mu^{\prime \prime}$ are monomials in the variables $\zeta_{i+1}, \zeta_{i+2}, \ldots, \zeta_{n}$. Since $\mathbb{D}_{k}^{t}$ is a normal subset of $\mathfrak{G}$ and since each $\alpha_{i, \sigma, j}$ is a singleton, it follows that $\operatorname{supp} \mu\left(\bar{x}, \bar{x}_{i+1}, \ldots, \bar{x}_{n}\right) \cap \mathbb{D}_{k}^{t} \neq \emptyset$ if and only if

$$
x \in\left(h^{\prime}\right)^{-1} \mathbb{D}_{k}^{t}\left(h^{\prime \prime}\right)^{-1}=\mathbb{D}_{k}^{t}\left(h^{\prime}\right)^{-1}\left(h^{\prime \prime}\right)^{-1}=\mathbb{D}_{k}^{t} y_{\mu}
$$


where supp $\mu^{\prime}\left(\bar{x}_{i+1}, \bar{x}_{i+2}, \ldots, \bar{x}_{n}\right)=\left\{h^{\prime}\right\}, \operatorname{supp} \mu^{\prime \prime}\left(\bar{x}_{i+1}, \bar{x}_{i+2}, \ldots, \bar{x}_{n}\right)=\left\{h^{\prime \prime}\right\}$, and $y_{\mu}=\left(h^{\prime}\right)^{-1}\left(h^{\prime \prime}\right)^{-1}$. In particular, if $x \in G \backslash T$ where

$$
T=\bigcup_{\mu \in \mathcal{N}_{i-1}} G \cap \mathbb{D}_{k}^{t} y_{\mu}
$$

then we have $\operatorname{supp} \mu\left(\bar{x}, \bar{x}_{i+1}, \ldots, \bar{x}_{n}\right) \cap \mathbb{D}_{k}^{t}=\emptyset$ for all $\mu \in \mathcal{N}_{i-1}=\mathcal{M}_{i-1} \backslash \mathcal{M}_{i}$ and hence for all $\mu \in \mathcal{M}_{i-1}$.

By induction, we conclude that

$$
\begin{aligned}
0 & =f_{i-1}\left(\bar{x}, \bar{x}_{i+1}, \ldots, \bar{x}_{n}\right) \\
& =\sum_{\sigma \in S_{i-1}} \sum_{j=1}^{a_{\sigma}} \beta_{i-1, \sigma, j} \bar{x}_{\sigma(i)} \alpha_{i, \sigma, j} \cdots \alpha_{n-1, \sigma, j} \bar{x}_{\sigma(n)} \alpha_{n, \sigma, j} \\
& \text { for all } x=x_{i} \in G \backslash T
\end{aligned}
$$

and we study this linear identity using Proposition 2.8. Specifically, we let the $\{\alpha, \beta\}$-terms correspond to those $\sigma \in S_{i}$ and the $\{\gamma, \delta\}$-terms correspond to those $\sigma \in S_{i-1} \backslash S_{i}$. Since all the summands in $f_{i-1}$ are singletons, it is clear, in the notation of the above mentioned proposition, that $r \geq 2 \max \{|A \cup C|,|B \cup D|\}$. Furthermore, since $f_{0}$ must contain at least two summands, it follows that $r \geq 4$. Thus, the hypotheses of Proposition 2.8 are satisfied, and there are two possible conclusions.

Suppose first that $|G: T| \leq(1 / r)^{3} \cdot 4^{r^{r+1}}$ !, and note that $T$ is a union of $\left|\mathcal{N}_{i-1}\right| \leq$ $|\mathcal{M}| \leq n r^{2}$ subsets of the form $G \cap \mathbb{D}_{k}^{t} y_{\mu}$ with $y_{\mu} \in \mathfrak{G}$. Furthermore, if $g, h \in$ $G \cap \mathbb{D}_{k}^{t} y_{\mu}$, then $g h^{-1} \in G \cap\left(\mathbb{D}_{k}^{t}\right)^{2} \subseteq G \cap \mathbb{D}_{k^{2}}^{t}=\Delta_{k^{2}}^{t}$. Thus $G \cap \mathbb{D}_{k}^{t} y_{\mu}$ is contained in a right $G$-translate of $\Delta_{k^{2}}^{t}$, and it follows from Lemma 2.1(ii) that $\left|G: \Delta_{k}^{t}\right| \leq$ $n r^{2} \cdot(1 / r)^{3} \cdot 4^{r^{r+1}} !=(n / r) \cdot 4^{r^{r+1}}$ !, contrary to our assumption. Consequently, the second possibility must occur and we consider that conclusion with $x=1$.

Observe that if $\sigma \in S_{i-1}$, then $\sigma \in S_{i}$ if and only if $\sigma(i)=i$ and hence if and only if

$$
\begin{aligned}
& \beta_{i-1, \sigma, j} \bar{x}_{\sigma(i)} \alpha_{i, \sigma, j} \cdots \alpha_{n-1, \sigma, j} \bar{x}_{\sigma(n)} \alpha_{n, \sigma, j} \\
& =\beta_{i-1, \sigma, j} \bar{x} \alpha_{i, \sigma, j} \cdots \alpha_{n-1, \sigma, j} \bar{x}_{\sigma(n)} \alpha_{n, \sigma, j}
\end{aligned}
$$

starts with $\beta_{i-1, \sigma, j} \bar{x} \alpha_{i, \sigma, j}$. Thus, since

$$
\begin{aligned}
\phi^{t}\left(\beta_{i-1, \sigma, j}\right) \alpha_{i, \sigma, j} & =\phi^{t}\left(\phi^{t}\left(\alpha_{0, \sigma, j}\right) \phi^{t}\left(\alpha_{1, \sigma, j}\right) \cdots \phi^{t}\left(\alpha_{i-2, \sigma, j}\right) \alpha_{i-1, \sigma, j}\right) \alpha_{i, \sigma, j} \\
& =\phi^{t}\left(\alpha_{0, \sigma, j}\right) \phi^{t}\left(\alpha_{1, \sigma, j}\right) \cdots \phi^{t}\left(\alpha_{i-2, \sigma, j}\right) \phi^{t}\left(\alpha_{i-1, \sigma, j}\right) \alpha_{i, \sigma, j} \\
& =\beta_{i, \sigma, j},
\end{aligned}
$$

it follows that the $\{\alpha, \beta\}$-terms (with $x=1$ ) in the conclusion of Proposition 2.8 sum to $f_{i}\left(\bar{x}_{i+1}, \bar{x}_{i+2}, \ldots, \bar{x}_{n}\right)$.

Next, note that a typical $\gamma$ term looks like

$$
\gamma=\beta_{i-1, \sigma, j} \bar{x}_{\sigma(i)} \alpha_{i, \sigma, j} \bar{x}_{\sigma(i+1)} \alpha_{i+1, \sigma, j} \cdots \alpha_{\ell-2, \sigma, j} \bar{x}_{\sigma(\ell-1)} \alpha_{\ell-1, \sigma, j}
$$

with $\sigma(\ell)=i$ and with $\ell \neq i$ since $\sigma \in S_{i-1} \backslash S_{i}$. Futhermore, if $y \in A \cap \mathbb{D}^{t}$, then certainly $\bar{y}=d \beta_{i-1, \tau, j^{\prime}}$ for some $\tau \in S_{i}, 1 \leq j^{\prime} \leq a_{\tau}$ and $0 \neq d \in K$. Thus $\psi(\gamma)$ is either equal to 0 or to a suitable

$$
\bar{y} \phi_{k^{\prime}}^{t}\left(\bar{y}^{-1} \gamma\right)=\beta_{i-1, \tau, j^{\prime}} \phi_{k^{\prime}}^{t}\left(\beta_{i-1, \tau, j^{\prime}}^{-1} \gamma\right)=\beta_{i-1, \tau, j^{\prime}} \phi_{k^{\prime}}^{t}\left(\mu\left(\bar{x}_{i+1}, \bar{x}_{i+2}, \ldots, \bar{x}_{n}\right)\right)
$$


with $k^{\prime} \leq k$ and with

$$
\begin{aligned}
\mu & =\beta_{i-1, \tau, j^{\prime}}^{-1} \gamma \\
& =\beta_{i-1, \tau, j^{\prime}}^{-1} \beta_{i-1, \sigma, j} \bar{x}_{\sigma(i)} \alpha_{i, \sigma, j} \bar{x}_{\sigma(i+1)} \alpha_{i+1, \sigma, j} \cdots \alpha_{\ell-2, \sigma, j} \bar{x}_{\sigma(\ell-1)} \alpha_{\ell-1, \sigma, j} \\
& \in \mathcal{M}_{i}^{\prime} \subseteq \mathcal{M}_{i} .
\end{aligned}
$$

But, by assumption, we have $\operatorname{supp} \mu\left(\bar{x}_{i+1}, \bar{x}_{i+2}, \ldots, \bar{x}_{n}\right) \cap \mathbb{D}_{k}^{t}=\emptyset$ and consequently $\phi_{k^{\prime}}^{t}\left(\mu\left(\bar{x}_{i+1}, \bar{x}_{i+2}, \ldots, \bar{x}_{n}\right)\right)=0$. Thus, again $\psi(\gamma)=0$, and it follows that the $\{\gamma, \delta\}$-terms contribute nothing to the sum in the conclusion of Proposition 2.8. In other words, that result yields $f_{i}\left(\bar{x}_{i+1}, \bar{x}_{i+2}, \ldots, \bar{x}_{n}\right)=0$, and the inductive step is proved.

In particular, when $i=n$, the inductive result implies that either $f_{n}=0$ or there exists $\mu \in \mathcal{M}_{n}$ with supp $\mu \cap \mathbb{D}_{k}^{t} \neq \emptyset$. However, $f_{n}$ is known to be a nonzero constant and $\mathcal{M}_{n}=\emptyset$. Hence we have a contradiction and therefore it follows that $\left|G: \Delta_{k}^{t}\right| \leq(n / r) \cdot 4^{r^{r+1}}$ !, as required.

Next, we isolate the procedure of [7, Lemma 2.1] which really only uses the fact that $G$ and $\mathbb{D}^{t}$ are normal in $\mathfrak{G}$.

Lemma 3.2. If $f_{1}(1,1, \ldots, 1) \neq 0$, then there exist $z_{1}, z_{2}, \ldots, z_{n} \in \mathfrak{G}$ so that

$$
\begin{aligned}
\tilde{f}\left(\zeta_{1}, \zeta_{2}, \ldots, \zeta_{n}\right) & =f\left(\bar{z}_{1}^{-1} \zeta_{1} \bar{z}_{1}, \bar{z}_{2}^{-1} \zeta_{2} \bar{z}_{2}, \ldots, \bar{z}_{n}^{-1} \zeta_{n} \bar{z}_{n}\right) \\
& =\sum_{\sigma \in \operatorname{Sym}_{n}} \sum_{j=1}^{a_{\sigma}} \tilde{\alpha}_{0, \sigma, j} \zeta_{\sigma(1)} \tilde{\alpha}_{1, \sigma, j} \zeta_{\sigma(2)} \cdots \tilde{\alpha}_{n-1, \sigma, j} \zeta_{\sigma(n)} \tilde{\alpha}_{n, \sigma, j}
\end{aligned}
$$

is a multilinear generalized polynomial identity for $K^{t}[G]$ with

$$
\sum_{j=1}^{a_{1}} \phi^{t}\left(\tilde{\alpha}_{0,1, j}\right) \phi^{t}\left(\tilde{\alpha}_{1,1, j}\right) \cdots \phi^{t}\left(\tilde{\alpha}_{n, 1, j}\right) \neq 0 .
$$

Proof. Let $Y$ be a right transversal for $\mathbb{D}^{t}$ in $\mathfrak{G}$. Then we can write each $\alpha_{i, 1, j}$ as $\alpha_{i, 1, j}=\sum_{s} \beta_{i, j, s} \bar{y}_{s}$ with $\beta_{i, j, s} \in K^{t}\left[\mathbb{D}^{t}\right]$ and $y_{s} \in Y$. Since

$$
0 \neq f_{1}(1,1, \ldots, 1)=\sum_{j=1}^{a_{1}} \alpha_{0,1, j} \alpha_{1,1, j} \cdots \alpha_{n, 1, j}
$$

and since each $\alpha_{i, 1, j}=\sum_{s} \beta_{i, j, s} \bar{y}_{s}$, there exist $y_{s_{0}}, y_{s_{1}}, \ldots, y_{s_{n}} \in Y$ with

$$
0 \neq \sum_{j=1}^{a_{1}} \beta_{0, j, s_{0}} \bar{y}_{s_{0}} \beta_{1, j, s_{1}} \bar{y}_{s_{1}} \cdots \beta_{n, j, s_{n}} \bar{y}_{s_{n}} .
$$

In particular, if we set $\bar{z}_{i}=\bar{y}_{s_{0}} \bar{y}_{s_{1}} \cdots \bar{y}_{s_{i-1}}$ and $\bar{z}_{0}=1$, then

$$
0 \neq \sum_{j=1}^{a_{1}}\left(\beta_{0, j, s_{0}}\right)^{\bar{z}_{0}^{-1}}\left(\beta_{1, j, s_{1}}\right)^{\bar{z}_{1}^{-1}} \cdots\left(\beta_{n, j, s_{n}}\right)^{\bar{z}_{n}^{-1}}
$$

Now $\beta_{i, j, s_{i}}=\phi^{t}\left(\alpha_{i, 1, j} \bar{y}_{s_{i}}^{-1}\right)$ so, since $\mathbb{D}^{t} \triangleleft \mathfrak{G}$,

$$
\left(\beta_{i, j, s_{i}}\right)^{\bar{z}_{i}^{-1}}=\phi^{t}\left(\bar{z}_{i} \alpha_{i, 1, j} \bar{y}_{s_{i}}^{-1} \bar{z}_{i}^{-1}\right)=\phi^{t}\left(\bar{z}_{i} \alpha_{i, 1, j} \bar{z}_{i+1}^{-1}\right) .
$$

It therefore follows that if we replace $\zeta_{i}$ in $f$ by $\bar{z}_{i}^{-1} \zeta_{i} \bar{z}_{i}$ and if we multiply $f$ on the right by $\bar{z}_{n+1}^{-1}$, then this new multilinear generalized polynomial $\tilde{f}$ has the required 
property concerning $\phi^{t}$. Furthermore, since $G$ is normal in $\mathfrak{G}, \tilde{f}$ is also an identity for $K^{t}[G]$.

With this, we now quickly prove our main result.

Theorem 3.3. Let $K^{t}[\mathfrak{G}]$ be a twisted group algebra of $\mathfrak{G}$ over $K$ and let $G$ be a normal subgroup of $\mathfrak{G}$. Suppose that $K^{t}[G]$ satisfies the multilinear generalized polynomial identity

$$
f\left(\zeta_{1}, \zeta_{2}, \ldots, \zeta_{n}\right)=\sum_{\sigma \in \operatorname{Sym}_{n}} \sum_{j=1}^{a_{\sigma}} \alpha_{0, \sigma, j} \zeta_{\sigma(1)} \alpha_{1, \sigma, j} \zeta_{\sigma(2)} \cdots \alpha_{n-1, \sigma, j} \zeta_{\sigma(n)} \alpha_{n, \sigma, j}
$$

with each $\alpha_{i, \sigma, j}$ in $K^{t}[\mathfrak{G}]$, but that there exists $\tau \in \operatorname{Sym}_{n}$ with $f_{\tau}$ not an identity for $K^{t}[G]$. If

$$
r=2 \sum_{\sigma \in \mathrm{Sym}_{n}} \sum_{j=1}^{a_{\sigma}} \prod_{i=0}^{n}\left|\operatorname{supp} \alpha_{i, \sigma, j}\right|,
$$

then $\left|G: \Delta_{k^{2}}^{t}\right| \leq 4^{r^{r+1}}$ ! where $k=4^{r^{r}}$.

Proof. We modify the identity $f$ so that Lemma 3.1 can apply. To start with, write each $\alpha_{i, \sigma, j}$ in terms of the $K$-basis $\overline{\mathfrak{G}}$ and apply the distributive law to each product

$$
\alpha_{0, \sigma, j} \zeta_{\sigma(1)} \alpha_{1, \sigma, j} \zeta_{\sigma(2)} \cdots \alpha_{n-1, \sigma, j} \zeta_{\sigma(n)} \alpha_{n, \sigma, j}
$$

By doing this, we obtain $\prod_{i=0}^{n}\left|\operatorname{supp} \alpha_{i, \sigma, j}\right|$ similar products but with parameters which are all singletons. In other words, we can now assume that each $\alpha_{i, \sigma, j}$ is a singleton and that $r / 2$ is equal to the total number of terms that occur. Indeed, if we choose to combine terms which are $K$-multiples of each other, then $r / 2$ will be greater than or equal to this total number of terms.

Next, we know that $f_{\tau}$ is not an identity for $K^{t}[G]$ and, by suitably permuting the variables, we can clearly assume that $\tau=1$. Furthermore, since $f_{1}$ is multilinear and since $\bar{G}$ is a $K$-basis for $K^{t}[G]$, there exist $g_{1}, g_{2}, \ldots, g_{n} \in G$ with $f_{1}\left(\bar{g}_{1}, \bar{g}_{2}, \ldots, \bar{g}_{n}\right) \neq 0$. Thus, by replacing each $\zeta_{i}$ by $\zeta_{i} \bar{g}_{i}$, which does not effect the number of terms in $f$ or the fact that each $\alpha_{i, \sigma, j}$ is a singleton, we can assume that $f_{1}(1,1, \ldots, 1) \neq 0$. Of course, this new $f$ is still an identity for $K^{t}[G]$.

Now for each $1 \neq \sigma \in \operatorname{Sym}_{n}$ for which a $\sigma$-term occurs in $f$, choose $i_{\sigma}, j_{\sigma} \in$ $\{1,2, \ldots, n\}$ such that $i_{\sigma}<j_{\sigma}$ but $\sigma\left(i_{\sigma}\right)>\sigma\left(j_{\sigma}\right)$. Let $U \subseteq\{1,2, \ldots, n\}$ be the collection of all such $i_{\sigma}$ and $j_{\sigma}$, and note that $|U| \leq 2 \cdot(r / 2)=r$ since $f$ has at most $r / 2$ terms. Suppose $f^{\prime}$ is the polynomial obtained from $f$ by setting $\zeta_{i}=1$ for all $i \notin U$. Then $f^{\prime}$ is certainly a multilinear generalized polynomial identity for $K^{t}[G]$, all its parameters are singletons, it has at most $r / 2$ terms, and $\operatorname{deg} f^{\prime}=|U| \leq r$. Furthermore, since $U$ contains all $i_{\sigma}$ and $j_{\sigma}$, we see that, if the $\zeta_{i}$-terms in

$$
\alpha_{0, \sigma, j} \zeta_{\sigma(1)} \alpha_{1, \sigma, j} \zeta_{\sigma(2)} \cdots \alpha_{n-1, \sigma, j} \zeta_{\sigma(n)} \alpha_{n, \sigma, j}
$$

are not in their natural order, then the truncated set of $\zeta_{i}$ 's which remain in $f^{\prime}$ are also not in their natural order. In other words, $f_{1}^{\prime}(1,1, \ldots, 1)=f_{1}(1,1, \ldots, 1) \neq 0$ and $f^{\prime}$ is a nondegenerate multilinear identity for $K^{t}[G]$. Since $\operatorname{deg} f^{\prime} \leq r$, this now allows us to assume that $n=\operatorname{deg} f \leq r$.

Finally, we apply Lemma 3.2 to $f$, and note that this does not change the degree of $f$, the number of terms it comprises, or the fact that each $\alpha_{i, \sigma, j}$ is a singleton. With this last modification, all the hypotheses of Lemma 3.1 are now satisfied, and since $n \leq r$, Lemma 3.1 yields the result. 
The remainder of the argument is entirely group theoretic.

Corollary 3.4. Let $K^{t}[\mathfrak{G}]$ be a twisted group algebra of $\mathfrak{G}$ over $K$ and let $G$ be a normal subgroup of $\mathfrak{G}$. Suppose that $K^{t}[G]$ satisfies the multilinear generalized polynomial identity

$$
f\left(\zeta_{1}, \zeta_{2}, \ldots, \zeta_{n}\right)=\sum_{\sigma \in \operatorname{Sym}_{n}} \sum_{j=1}^{a_{\sigma}} \alpha_{0, \sigma, j} \zeta_{\sigma(1)} \alpha_{1, \sigma, j} \zeta_{\sigma(2)} \cdots \alpha_{n-1, \sigma, j} \zeta_{\sigma(n)} \alpha_{n, \sigma, j}
$$

with each $\alpha_{i, \sigma, j}$ in $K^{t}[\mathfrak{G}]$, but that there exists $\tau \in \operatorname{Sym}_{n}$ with $f_{\tau}$ not an identity for $K^{t}[G]$. If

$$
r=2 \sum_{\sigma \in \operatorname{Sym}_{n}} \sum_{j=1}^{a_{\sigma}} \prod_{i=0}^{n}\left|\operatorname{supp} \alpha_{i, \sigma, j}\right|
$$

then

(i) $|G: \Delta| \leq 4^{r^{r+1}}$ ! and the commutator subgroup of $\Delta$ is finite.

(ii) $\left|G: \Delta^{t}\right| \leq 4^{r^{r+1}}$ ! and the commutator subgroup of $\Delta^{t}$ is finite.

(iii) $G$ has a normal subgroup $H \subseteq \Delta^{t}$ with $|G: H| \leq 4^{r^{r+1}}$ ! and with $\left|H^{\prime}\right|$ bounded by a function of $r$. Furthermore, $H=\Delta_{h}^{t}(H)$ for some integer $h$ depending upon $r$.

Proof. If $k=4^{r^{r}}$, then the previous theorem implies that $\left|G: \Delta_{k^{2}}^{t}\right| \leq 4^{r^{r+1}}$ ! $=\ell$. In particular, since $\Delta \supseteq \Delta^{t} \supseteq \Delta_{k^{2}}^{t}$, it follows that $\ell$ also bounds $|G: \Delta|$ and $\left|G: \Delta^{t}\right|$. Furthermore, since $\Delta_{k^{2}} \supseteq \Delta_{k^{2}}^{t}$, we have $\left|G: \Delta_{k^{2}}\right|<\infty$ and [7, Lemma 2.3] implies that the commutator subgroup of $\Delta$ is finite. Clearly, the same is also true of the commutator subgroup of $\Delta^{t} \subseteq \Delta$.

Finally, we let $H$ be the subgroup of $G$ generated by $\Delta_{k^{2}}^{t}$. Then $H \triangleleft G, H \subseteq \Delta^{t}$, and $|G: H| \leq\left|G: \Delta_{k^{2}}^{t}\right| \leq \ell$. Since $H=\left(\Delta_{k^{2}}^{t}\right)^{4^{\ell}}$, by [8, Lemma 5.2.3], it follows that $H=\Delta_{h}^{t}(H)$ where $h=\left(k^{2}\right)^{4^{\ell}}$. In particular, we also have $H=\Delta_{h}(H)$, so [8, Theorem 5.2.9], a result of Wiegold, implies that $\left|H^{\prime}\right|$ is bounded by a function of $h$ and hence by a function of $r$.

Theorem 1.1 is, of course, an immediate consequence of part (iii) above and, in some sense, it is the most that we can conclude about the group-theoretic structure of $G$. Indeed, it was shown in [7] that an ordinary group algebra $K[G]$ satisfies a nondegenerate multilinear polynomial identity if and only if $G$ has a normal subgroup $H$ of finite index with $H^{\prime}$ finite. But this structure does not guarantee a g.p.i. in the case of twisted group algebras as can be seen in the following

Example 3.5. Let $p$ be a prime and let $K$ be a field containing a nonidentity $p$ th root of unity. If $G$ is a countably infinite elementary abelian $p$-group, then there exists a twisted group algebra $K^{t}[G]$ which does not satisfy a nondegenerate multilinear generalized polynomial identity, but does have $G=\Delta_{p}^{t}(G)$.

Proof. Let $\varepsilon \neq 1$ be the given $p$ th root of unity in $K$, and let $x_{1}, x_{2}, \ldots, y_{1}, y_{2}, \ldots$ be a basis for $G$. Define $R$ to be the $K$-algebra whose generators $\bar{x}_{1}, \bar{x}_{2}, \ldots, \bar{y}_{1}, \bar{y}_{2}, \ldots$ satisfy the relations $\bar{x}_{i}^{p}=1=\bar{y}_{i}^{p}, \bar{x}_{i} \bar{x}_{j}=\bar{x}_{j} \bar{x}_{i}, \bar{y}_{i} \bar{y}_{j}=\bar{y}_{j} \bar{y}_{i}$, and

$$
\bar{x}_{i} \bar{y}_{j}= \begin{cases}\bar{y}_{j} \bar{x}_{i}, & \text { if } i \neq j, \text { or } \\ \varepsilon \bar{y}_{j} \bar{x}_{i}, & \text { if } i=j .\end{cases}
$$


Then it is easy to see that $R=K^{t}[G]$ is a twisted group algebra of $G$. In fact, if $g \in G$, then the possible $\bar{G}$-conjugates of $\bar{g}$ are $\bar{g}, \varepsilon \bar{g}, \ldots, \varepsilon^{p-1} \bar{g}$, so $g \in \Delta_{p}^{t}(G)$, and hence $G=\Delta_{p}^{t}(G)$.

Note that the subalgebra of $K^{t}[G]$ generated by $\bar{x}_{i}, \bar{y}_{i}$ is isomorphic to the matrix ring $\mathrm{M}_{p}(K)$, since this corresponds to a nonlinear irreducible representation of a nonabelian group of order $p^{3}$. Thus, the subalgebra of $K^{t}[G]$ generated by $k$ pairs $\left\{\bar{x}_{i}, \bar{y}_{i}\right\}$ is isomorphic to $\mathrm{M}_{p}(K)^{\otimes k}=\mathrm{M}_{p^{k}}(K)$.

Finally, suppose that $K^{t}[G]$ satisfies the nondegenerate g.p.i.

$$
f\left(\zeta_{1}, \zeta_{2}, \ldots, \zeta_{n}\right)=\sum_{\sigma \in \operatorname{Sym}_{n}} f_{\sigma}\left(\zeta_{1}, \zeta_{2}, \ldots, \zeta_{n}\right)
$$

and let $s_{1}, s_{2}, \ldots, s_{n} \in K^{t}[G]$ with $f_{1}\left(s_{1}, s_{2}, \ldots, s_{n}\right) \neq 0$. We may assume that all terms in $f\left(\zeta_{1}, \zeta_{2}, \ldots, \zeta_{n}\right)$ and all $s_{1}, s_{2}, \ldots, s_{n}$ are contained in $K^{t}[H]$, where $H=$ $\left\langle x_{1}, y_{1}, x_{2}, y_{2}, \ldots, x_{r}, y_{r}\right\rangle$. Now let $W=\left\langle x_{r+1}, y_{r+1}, x_{r+2}, y_{r+2}, \ldots, x_{r+k}, y_{r+k}\right\rangle$ so that $K^{t}[W] \cong \mathrm{M}_{p^{k}}(K)$. Furthermore, since $K^{t}[W]$ and $K^{t}[H]$ commute elementwise, we see that $K^{t}[W]$ satisfies the identity

$$
f\left(s_{1} \zeta_{1}, s_{2} \zeta_{2}, \ldots, s_{n} \zeta_{n}\right)=\sum_{\sigma \in \operatorname{Sym}_{n}} f_{\sigma}\left(s_{1}, s_{2}, \ldots, s_{n}\right) \zeta_{\sigma(1)} \zeta_{\sigma(2)} \cdots \zeta_{\sigma(n)} .
$$

In particular, if $k$ is chosen so that $p^{k}>n$, then we can take $\zeta_{1}, \zeta_{2}, \ldots, \zeta_{n}$ to be the matrix units $\zeta_{i}=e_{i, i+1}$ and conclude that

$$
f\left(s_{1} e_{1,2}, s_{2} e_{2,3}, \ldots, s_{n} e_{n, n+1}\right)=f_{1}\left(s_{1}, s_{2}, \ldots, s_{n}\right) e_{1, n+1}
$$

since all products of the matrix units other than $e_{1,2} e_{2,3} \cdots e_{n, n+1}=e_{1, n+1}$ are zero. But $0 \neq f_{1}\left(s_{1}, s_{2}, \ldots, s_{n}\right) \in K^{t}[H]$, and $0 \neq e_{1, n+1} \in K^{t}[W]$, so we have a contradiction since $K^{t}[H \times W]=K^{t}[H] \otimes K^{t}[W]$.

In the preceding example, if $H$ is any subgroup of $G$ of finite index, then it is easy to see that $K^{t}[H]$ is noncommutative. On the other hand, $G$ is an abelian group, so the ordinary group algebra $K[H]$ is certainly commutative. In particular, the two algebras $K^{t}[H]$ and $K[H]$ are not $K$-isomorphic, nor can they become isomorphic under any field extension of $K$. As we will see in the next section, it is precisely this condition which keeps $K^{t}[G]$ from satisfying a nondegenerate multilinear polynomial identity.

\section{Stable Untwisting}

We say that the twisted group algebra $K^{t}[G]$ is untwisted if there exists a function $G \rightarrow K^{\bullet}$, given by $x \mapsto d_{x}$, such that the elements $\tilde{x}=d_{x} \bar{x}$ with $x \in G$ form a group basis for $K^{t}[G]$. Thus $\tilde{x} \tilde{y}=\widetilde{x y}$ for all $x, y \in G$, and $K^{t}[G] \cong K[G]$ via this diagonal change of basis. In addition, we say that $K^{t}[G]$ is stably untwisted if there exists a field $F \supseteq K$ such that $F^{t}[G]=F \otimes_{K} K^{t}[G]$ is untwisted. The standard untwisting criterion is

Lemma 4.1. $K^{t}[G]$ is untwisted if and only if there exists an algebra homomorphism $\rho: K^{t}[G] \rightarrow K$. Furthermore, $K^{t}[G]$ is stably untwisted if and only if there exists an algebra homomorphism $\rho: K^{t}[G] \rightarrow R$ with $R$ a commutative algebra.

Proof. If $K^{t}[G] \cong K[G]$, then there exists a homomorphism $\rho: K^{t}[G] \rightarrow K$ corresponding to the augmentation map of $K[G]$. Conversely, if $\rho$ exists, we define $\tilde{x}=\rho(x)^{-1} \bar{x}$ for all $x \in G$. Then $\tilde{x}$ is the unique element in $K \bar{x}$ with $\rho(\tilde{x})=1$, and 
consequently $\tilde{x} \tilde{y}=\widetilde{x y}$ for all $x, y \in G$. Thus $\widetilde{G}=\{\tilde{x} \mid x \in G\}$ is a group basis for $K^{t}[G]$, and $K^{t}[G] \cong K[G]$.

If $K^{t}[G]$ is stably untwisted with associated field $F \supseteq K$, then by the above we have an algebra homomorphism $\rho: K^{t}[G] \rightarrow F^{t}[G] \rightarrow F$. Conversely, if $\rho$ exists, let $F$ be an algebraically closed field extension of $K$ with $|F|>|G|$, an inequality of cardinal numbers. Then $\rho$ extends to an $F$-algebra map $\rho: F^{t}[G]=F \otimes_{K} K^{t}[G] \rightarrow$ $F \otimes_{K} R \rightarrow L$ where the field $L$ is equal to $F \otimes R$ modulo a maximal ideal. Since $\operatorname{dim}_{F} L \leq \operatorname{dim}_{F} F^{t}[G]=|G|<|F|$, [8, Lemma 7.1.2(iii)] implies that $L=F$. Thus $F^{t}[G]$ is untwisted and $K^{t}[G]$ is stably untwisted.

As an application of the above, suppose $a \in K$ does not have an $n$th root in the field, and let $G=\langle x\rangle$ be cyclic of order $n$. Then $K^{t}[G]=K\left[\bar{x} \mid \bar{x}^{n}=a\right]$ cannot be untwisted over $K$, but it can be untwisted once we adjoin an $n$th root of $a$ to the field. Next, we show

Lemma 4.2. Let $K^{t}[G]$ be given and let $H$ be a normal subgroup of $G$ of finite index with $\left|H^{\prime}\right|<\infty$ and with $K^{t}[H]$ stably untwisted. Then $K^{t}[G]$ satisfies a nondegenerate multilinear generalized polynomial identity.

Proof. By assumption, there exists a field $F \supseteq K$ with $F \otimes_{K} K^{t}[H]=F^{t}[H]$ untwisted, so that $F^{t}[H] \cong F[H]$. Define $I$ to be the characteristic ideal of $F^{t}[H]$ generated by all Lie products $[\gamma, \delta]=\gamma \delta-\delta \gamma$ with $\gamma, \delta \in F^{t}[H]$. Thus $I$ is the smallest ideal of $F^{t}[H]$ with commutative quotient. Viewed in $F[H]$, we see that $I \subseteq \omega\left(F\left[H^{\prime}\right]\right) \cdot F[H]$, the kernel of the natural map $F[H] \rightarrow F\left[H / H^{\prime}\right]$, and hence, since $H^{\prime}$ is finite, we have $\alpha I=0$ where $\alpha \neq 0$ is the sum in $F\left[H^{\prime}\right]$ of all elements of $H^{\prime}$. In other words, there exists $0 \neq \alpha \in F^{t}\left[H^{\prime}\right] \subseteq F^{t}[H]$ with $\alpha I=0$.

Next, let $|G: H|=n$, write $M=\mathrm{M}_{n}\left(F^{t}[H]\right)$ and consider the natural homomorphism $\mathrm{M}_{n}\left(F^{t}[H]\right) \rightarrow \mathrm{M}_{n}\left(F^{t}[H] / I\right)$. Since $F^{t}[H] / I$ is commutative, the AmitsurLevitzki theorem (see $\left[8\right.$, Theorem 5.1.9]) implies that $\mathrm{M}_{n}\left(F^{t}[H] / I\right.$ ) satisfies the standard identity

$$
s_{m}\left(\zeta_{1}, \zeta_{2}, \ldots, \zeta_{m}\right)=\sum_{\sigma \in \operatorname{Sym}_{m}}(-1)^{\sigma} \zeta_{\sigma(1)} \zeta_{\sigma(2)} \cdots \zeta_{\sigma(m)}
$$

with $m=2 n$. In particular, we have $s_{m}(M, M, \ldots, M) \subseteq \mathrm{M}_{n}(I)$.

Let $\left\{x_{1}, x_{2}, \ldots, x_{n}\right\}$ be a transversal for $H$ in $G$, and use it to construct an embedding of $F^{t}[G]$ into $M=\mathrm{M}_{n}\left(F^{t}[H]\right)$ as in [8, Lemma 5.1.10]. Then under this embedding, $\alpha \in F^{t}[H]$ maps to the diagonal matrix

$$
\operatorname{diag}\left(\bar{x}_{1} \alpha \bar{x}_{1}^{-1}, \bar{x}_{2} \alpha \bar{x}_{2}^{-1}, \ldots, \bar{x}_{n} \alpha \bar{x}_{n}^{-1}\right),
$$

and note that each $\bar{x}_{i} \alpha \bar{x}_{i}^{-1}$ annihilates $I$ since $\alpha$ annihilates $I$ and since conjugation by $\bar{x}_{i}^{-1}$ is an automorphism of $F^{t}[H]$ stabilizing the characteristic ideal $I$. With this, we conclude that $\alpha s_{m}\left(F^{t}[G], F^{t}[G], \ldots, F^{t}[G]\right)$ maps to 0 under the embedding into $M$, and hence we see that $F^{t}[G]$ satisfies the multilinear generalized polynomial identity

$$
\alpha s_{m}\left(\zeta_{1}, \zeta_{2}, \ldots, \zeta_{m}\right)=\sum_{\sigma \in \operatorname{Sym}_{m}}(-1)^{\sigma} \alpha \zeta_{\sigma(1)} \zeta_{\sigma(2)} \cdots \zeta_{\sigma(m)} .
$$

Finally, since $F^{t}[G]$ is free over $K^{t}[G]$, we conclude that $K^{t}[G]$ satisfies the multilinear generalized polynomial identity

$$
f\left(\zeta_{1}, \zeta_{2}, \ldots, \zeta_{m}\right)=\beta s_{m}\left(\zeta_{1}, \zeta_{2}, \ldots, \zeta_{m}\right)=\sum_{\sigma \in \operatorname{Sym}_{m}}(-1)^{\sigma} \beta \zeta_{\sigma(1)} \zeta_{\sigma(2)} \cdots \zeta_{\sigma(m)}
$$


where $0 \neq \beta \in K\left[H^{\prime}\right]$ occurs in the representation of $\alpha \in F\left[H^{\prime}\right]=F \otimes_{K} K\left[H^{\prime}\right]$. Of course, $f$ is nondegenerate since $f_{1}(1,1, \ldots, 1)=\beta \neq 0$.

As we will see, there is a converse to Lemma 4.2 which holds in the more general context of $G \subseteq \mathfrak{G}$. To start with, we need the following standard fact.

Lemma 4.3. Let $K^{t}[G]$ be a twisted group algebra of the abelian group $G$ and suppose that $K^{t}[G]$ satisfies an ordinary polynomial identity of degree $n$. Then $G$ has a subgroup $Z$ of index $\leq(n / 2)^{2}$ with $K^{t}[Z]$ central in $K^{t}[G]$.

Proof. Let $F$ be an algebraically closed field extension of $K$ with $|F|>|G|$, and observe that $F^{t}[G]=F \otimes_{K} K^{t}[G]$ also satisfies an ordinary polynomial identity of degree $n$. In particular, if $\xi: F^{t}[G] \rightarrow R$ is any irreducible representation, then $R$ is a primitive p.i. algebra, so Kaplansky's theorem (see [8, Theorem 5.3.4]) implies that $R \cong \mathrm{M}_{m}(D)$ for some $F$-division algebra $D$ and some integer $m \leq n / 2$. Furthermore, $\operatorname{dim}_{F} D \leq \operatorname{dim}_{F} F^{t}[G]=|G|<|F|$ and hence, by [8, Lemma 7.1.2(iii)] again, $D=F$. Let $Z=\left\{g \in G \mid \bar{g}\right.$ is central in $\left.K^{t}[G]\right\}$. Then $Z$ is a subgroup of $G$ and $K^{t}[Z]$ is central in $K^{t}[G]$.

Now define $\chi: G \rightarrow F$ so that, for each $g \in G, \chi(g)$ is the trace of the $m \times m$ matrix $\xi(\bar{g})$. If $g \in Z$, then $\xi(\bar{g})$ is central in $\mathrm{M}_{m}(F)$ and hence it is a scalar matrix. Thus $\xi(\bar{g}) \in F$ and $\chi(g)=m \cdot \xi(\bar{g})$. On the other hand, if $g \in G \backslash Z$, then there exists $h \in G$ so that $\bar{g}$ and $\bar{h}$ do not commute. In particular, since $G$ is abelian, we have $\bar{h}^{-1} \bar{g} \bar{h}=k \bar{g}$ for some $1 \neq k \in K^{\bullet}$, and then $\xi(\bar{h})^{-1} \xi(\bar{g}) \xi(\bar{h})=k \cdot \xi(\bar{g})$. But similar matrices have the same trace, so $\chi(g)=k \chi(g)$ and $\chi(g)=0$ since $k \neq 1$. It follows that $m \neq 0$ in $F$, since $\xi(\bar{G})$ spans $\mathrm{M}_{m}(F)$ and since there exist matrices with nonzero trace.

Finally, if $\left\{g_{1}, g_{2}, \ldots\right\}$ is a transversal for $Z$ in $G$, then we claim that the set $\left\{\xi\left(\bar{g}_{1}\right), \xi\left(\bar{g}_{2}\right), \ldots\right\}$ is $F$-linearly independent in $\mathrm{M}_{m}(F)$. Indeed, if $\sum_{j=1}^{s} f_{j} \xi\left(\bar{g}_{j}\right)=0$ is an $F$-linear relation, then for all $i$ we have $\sum_{j=1}^{s} f_{j} \xi\left(\bar{g}_{j}\right) \xi\left(\bar{g}_{i}\right)^{-1}=0$. Moreover, $g_{j, i}=g_{j} g_{i}^{-1} \in Z$ if and only if $i=j$, and in all cases $\bar{g}_{j} \bar{g}_{i}^{-1}$ is a scalar multiple of $\bar{g}_{j, i}$. Thus, by taking traces, we get $0=f_{i} \cdot \chi(1)=f_{i} \cdot m$, and hence $f_{i}=0$ since $m \neq 0$ in $F$. We conclude from this linear independence that $|G: Z| \leq \operatorname{dim}_{F} \mathrm{M}_{m}(F)=m^{2}$, and since $m \leq n / 2$, the lemma is proved.

Next, we need

Lemma 4.4. Suppose $K^{t}[G]$ is a commutative twisted group algebra of the finite group $G$ over the algebraically closed field $K$. If $\Omega$ is a nonempty, nonzero subset of $K^{t}[G]$, then there exists $\gamma \in K^{t}[G]$ with $0 \neq \gamma \Omega \subseteq I$, a 1-dimensional ideal.

Proof. Since $K$ is algebraically closed, and $K^{t}[G]$ is commutative and finite dimensional, it follows from Lemma 4.1 that $K^{t}[G]=K[G]$ is an ordinary group algebra of the finite abelian group $G$. Let char $K=p \geq 0$ and write $G=P \times H$ where $P$ is the Sylow $p$-subgroup of $G$ and $H$ is the $p$-complement. Of course, if $p=0$, then $G=H$ and $P=1$.

Since $K[H]$ is semiprimitive, write $K[H]=\sum_{1}^{t} e_{j} K$, where $\left\{e_{j} \mid j=1, \ldots, t\right\}$ is the set of primitive idempotents of this group algebra. Certainly, $e_{i} \Omega \neq 0$ for some $i$, and $e_{i} \Omega \subseteq e_{i} K[P] \cong K[P]$ since $K[G] \cong K[H] \otimes_{K} K[P]$. Finally, let $\omega(K[P])$ be the augmentation ideal of $K[P]$, and note that this ideal is nilpotent. Thus there exists an integer $m \geq 0$ maximal with $\omega(K[P])^{m} e_{i} \Omega \neq 0$, and we can choose $\gamma \in \omega(K[P])^{m} e_{i}$ with $\gamma \Omega \neq 0$. But then $\gamma \Omega$ is a subset of $e_{i} K[P] \cong K[P]$ 
annihilated by the augmentation ideal, so $\gamma \Omega \subseteq K e_{i} \hat{P}=I$, where $\hat{P}$ is the sum of the elements of $P$ in $K[G]$.

The above result actually relies upon the fact that $K[G]$ is a Frobenius algebra, so that each minimal ideal of this commutative algebra corresponds to a different irreducible representation. On the other hand, if $A=K\left[x, y \mid(x, y)^{2}=0\right]$, then it is clear that there is no multiple of $\Omega=\{x, y\}$ which is both nonzero and contained in a 1-dimensional subspace. Now we return to infinite groups.

Lemma 4.5. Let $K^{t}[\mathfrak{G}]$ be given and let $G$ be a normal subgroup of $\mathfrak{G}$. Suppose that $K^{t}[G]$ satisfies the multilinear generalized polynomial identity

$$
f\left(\zeta_{1}, \zeta_{2}, \ldots, \zeta_{n}\right)=\sum_{\sigma \in \operatorname{Sym}_{n}} \sum_{j=1}^{a_{\sigma}} \alpha_{0, \sigma, j} \zeta_{\sigma(1)} \alpha_{1, \sigma, j} \zeta_{\sigma(2)} \cdots \alpha_{n-1, \sigma, j} \zeta_{\sigma(n)} \alpha_{n, \sigma, j}
$$

with each $\alpha_{i, \sigma, j}$ in $K^{t}[\mathfrak{G}]$, and assume that there exists $\tau \in \operatorname{Sym}_{n}$ with $f_{\tau}$ not an identity for $K^{t}[G]$. Then $G$ has a subgroup $H$ of finite index with $K^{t}[H]$ satisfying an identity of the form

$$
\tilde{f}\left(\zeta_{1}, \zeta_{2}, \ldots, \zeta_{n}\right)=\sum_{\sigma \in \operatorname{Sym}_{n}} \alpha_{\sigma} \zeta_{\sigma(1)} \zeta_{\sigma(2)} \cdots \zeta_{\sigma(n)}
$$

with $\alpha_{\sigma} \in K^{t}[\mathfrak{G}]$ for all $\sigma \in \operatorname{Sym}_{n}$ and with $\alpha_{1} \neq 0$. Furthermore, $\left|H^{\prime}\right|<\infty$ and $K^{t}\left[H^{\prime}\right]$ is central in $K^{t}[H]$.

Proof. As in the proof of Theorem 3.3, we first modify $f$ so that $f_{1}(1,1, \ldots, 1) \neq 0$ and that all $\alpha_{i, \sigma, j}$ are singletons. Then we apply Lemma 3.2 to obtain a new identity

$$
f\left(\zeta_{1}, \zeta_{2}, \ldots, \zeta_{n}\right)=\sum_{\sigma \in \operatorname{Sym}_{n}} \sum_{j=1}^{a_{\sigma}} \alpha_{0, \sigma, j} \zeta_{\sigma(1)} \alpha_{1, \sigma, j} \zeta_{\sigma(2)} \cdots \alpha_{n-1, \sigma, j} \zeta_{\sigma(n)} \alpha_{n, \sigma, j}
$$

with

$$
\sum_{j=1}^{a_{1}} \phi^{t}\left(\alpha_{0,1, j}\right) \phi^{t}\left(\alpha_{1,1, j}\right) \cdots \phi^{t}\left(\alpha_{n, 1, j}\right) \neq 0
$$

while still preserving the fact that each $\alpha_{i, \sigma, j}$ is a singleton. Let $H=\Delta^{t}(G)$ so that $|G: H|<\infty$ and $\left|H^{\prime}\right|<\infty$ by Corollary 3.4(ii). Since $|G: H|<\infty$, it is clear that $\mathbb{D}_{\mathfrak{G}}^{t}(G)=\mathbb{D}_{\mathfrak{G}}^{t}(H)$, and hence $\phi^{t}$ above maps $K^{t}[\mathfrak{G}]$ to $K^{t}\left[\mathbb{D}_{\mathfrak{G}}^{t}(H)\right]$.

Fix $h_{2}, h_{3}, \ldots, h_{n} \in H$ and consider the linear identity

$$
\begin{aligned}
0 & =f\left(\bar{h}_{1}, \bar{h}_{2}, \ldots, \bar{h}_{n}\right) \\
& =\sum_{\sigma, j} \alpha_{0, \sigma, j} \bar{h}_{\sigma(1)} \alpha_{1, \sigma, j} \bar{h}_{\sigma(2)} \cdots \alpha_{n-1, \sigma, j} \bar{h}_{\sigma(n)} \alpha_{n, \sigma, j} \\
& =\sum_{\sigma, j} \beta_{\sigma, j} \bar{h}_{1} \gamma_{\sigma, j} \quad \text { for all } h_{1} \in H .
\end{aligned}
$$

Then, by Lemma 2.7 with $T=\emptyset$, we have $0=\sum_{\sigma, j} \phi^{t}\left(\beta_{\sigma, j}\right) \bar{h}_{1} \gamma_{\sigma, j}$ for all $h_{1} \in H$ and, by applying $\phi^{t}$ to this expression, we obtain

$$
0=\sum_{\sigma, j} \phi^{t}\left(\beta_{\sigma, j}\right) \bar{h}_{1} \phi^{t}\left(\gamma_{\sigma, j}\right) \quad \text { for all } h_{1} \in H
$$

since $\phi^{t}\left(\beta_{\sigma, j}\right) \bar{h}_{1} \in K\left[\mathbb{D}_{\mathfrak{G}}^{t}(H)\right]$. Note that each $\alpha_{i, \sigma, j}$ is a singleton, so it follows that each $\beta_{\sigma, j}$ and $\gamma_{\sigma, j}$ is also a singleton. Hence $\phi^{t}\left(\beta_{\sigma, j}\right)=0$ or $\beta_{\sigma, j}$, and $\phi^{t}\left(\gamma_{\sigma, j}\right)=0$ 
or $\gamma_{\sigma, j}$. Furthermore, since $H \subseteq \mathbb{D}_{\mathfrak{G}}^{t}(H)=\mathbb{D}_{\mathfrak{G}}^{t}(G) \triangleleft \mathfrak{G}$, the question of whether $\operatorname{supp} \beta_{\sigma, j} \in \mathbb{D}_{\mathfrak{G}}^{t}(H)$ or not does not depend upon the elements $\bar{h}_{2}, \bar{h}_{3}, \ldots, \bar{h}_{n}$ that occur in the product expression for $\beta_{\sigma, j}$, and similarly this is also true for $\gamma_{\sigma, j}$. Thus, if $\sum_{\sigma, j}^{\prime}$ denotes the partial sum of all those terms with $\phi^{t}\left(\beta_{\sigma, j}\right)=\beta_{\sigma, j}$ and $\phi^{t}\left(\gamma_{\sigma, j}\right)=\gamma_{\sigma, j}$, then we see that

$$
0=\sum_{\sigma, j}^{\prime} \beta_{\sigma, j} \bar{h}_{1} \gamma_{\sigma, j}
$$

is a multilinear identity that holds for all $h_{1}, h_{2}, \ldots, h_{n} \in H$.

We continue this process, applying the above truncation procedure to the variables $h_{2}, h_{3}, \ldots, h_{n}$ in turn, thereby obtaining a multilinear generalized polynomial

$$
f^{\prime \prime}\left(\zeta_{1}, \zeta_{2}, \ldots, \zeta_{n}\right)=\sum_{\sigma, j}^{\prime \prime} \alpha_{0, \sigma, j} \zeta_{\sigma(1)} \alpha_{1, \sigma, j} \zeta_{\sigma(2)} \cdots \alpha_{n-1, \sigma, j} \zeta_{\sigma(n)} \alpha_{n, \sigma, j}
$$

satisfied by $K^{t}[H]$. Again $\sum_{\sigma, j}^{\prime \prime}$ denotes the partial sum of all those terms that survive this process. We note that if

$$
\alpha_{0, \sigma, j} \zeta_{\sigma(1)} \alpha_{1, \sigma, j} \zeta_{\sigma(2)} \cdots \alpha_{n-1, \sigma, j} \zeta_{\sigma(n)} \alpha_{n, \sigma, j}
$$

survives, then when the truncation procedure was applied to $\bar{h}_{\sigma(1)}$ we must have had supp $\alpha_{0, \sigma, j} \in \mathbb{D}_{\mathfrak{G}}^{t}(H)$. Furthermore, when the procedure was applied to $\bar{h}_{\sigma(2)}$, we must have had $\operatorname{supp} \alpha_{0, \sigma, j} \alpha_{1, \sigma, j} \in \mathbb{D}_{\mathfrak{G}}^{t}(H)$, and hence $\operatorname{supp} \alpha_{1, \sigma, j} \in \mathbb{D}_{\mathfrak{G}}^{t}(H)$. Continuing in this manner, we see that

$$
\begin{aligned}
& f^{\prime \prime}\left(\zeta_{1}, \zeta_{2}, \ldots, \zeta_{n}\right) \\
& \quad=\sum_{\sigma, j} \phi^{t}\left(\alpha_{0, \sigma, j}\right) \zeta_{\sigma(1)} \phi^{t}\left(\alpha_{1, \sigma, j}\right) \zeta_{\sigma(2)} \cdots \phi^{t}\left(\alpha_{n-1, \sigma, j}\right) \zeta_{\sigma(n)} \phi^{t}\left(\alpha_{n, \sigma, j}\right),
\end{aligned}
$$

where the $\alpha_{n, \sigma, j}$ term comes from a consideration of the truncation procedure applied to $\bar{h}_{\sigma(n)}$.

If $A=\bigcup_{i, \sigma, j} \operatorname{supp} \phi^{t}\left(\alpha_{i, \sigma, j}\right)$, then $A$ is a finite subset of $\mathbb{D}_{\mathfrak{G}}^{t}(H)$, and hence $C=\bigcap_{a \in A} \mathbb{C}_{H}^{t}(\bar{a})$ is a subgroup of finite index in $H$. Furthermore, $K^{t}[C]$ satisfies the above identity and it centralizes all the factors $\phi^{t}\left(\alpha_{i, \sigma, j}\right)$. Thus we see that $K^{t}[C]$ satisfies

$$
\sum_{\sigma \in \operatorname{Sym}_{n}} \alpha_{\sigma} \zeta_{\sigma(1)} \zeta_{\sigma(2)} \cdots \zeta_{\sigma(n)}
$$

where

$$
\alpha_{\sigma}=\sum_{j} \phi^{t}\left(\alpha_{0, \sigma, j}\right) \phi^{t}\left(\alpha_{1, \sigma, j}\right) \cdots \phi^{t}\left(\alpha_{n-1, \sigma, j}\right) \phi^{t}\left(\alpha_{n, \sigma, j}\right) .
$$

In particular, we know that $\alpha_{1} \neq 0$.

Finally, $C \subseteq H$, so $C^{\prime} \subseteq H^{\prime}$ and $\left|C^{\prime}\right|<\infty$. Thus, since $C \subseteq H=\Delta^{t}(H)$, we see that $L=\mathbb{C}_{C}^{t}\left(C^{\prime}\right)$ is a subgroup of $C$ of finite index. Moreover, $L^{\prime} \subseteq C^{\prime}$, so $L=\mathbb{C}_{L}^{t}\left(L^{\prime}\right)$ and $K^{t}\left[L^{\prime}\right]$ is central in $K^{t}[L]$. With this, the result follows using $L$ as the appropriate subgroup of $G$.

We can now obtain the promised converse to Lemma 4.2.

Theorem 4.6. Let $K^{t}[\mathfrak{G}]$ be a twisted group algebra of $\mathfrak{G}$ over $K$ and let $G$ be a normal subgroup of $\mathfrak{G}$. Suppose that $K^{t}[G]$ satisfies the multilinear generalized polynomial identity

$$
f\left(\zeta_{1}, \zeta_{2}, \ldots, \zeta_{n}\right)=\sum_{\sigma \in \operatorname{Sym}_{n}} \sum_{j=1}^{a_{\sigma}} \alpha_{0, \sigma, j} \zeta_{\sigma(1)} \alpha_{1, \sigma, j} \zeta_{\sigma(2)} \cdots \alpha_{n-1, \sigma, j} \zeta_{\sigma(n)} \alpha_{n, \sigma, j}
$$


with each $\alpha_{i, \sigma, j}$ in $K^{t}[\mathfrak{G}]$, but that there exists $\tau \in \operatorname{Sym}_{n}$ with $f_{\tau}$ not an identity for $K^{t}[G]$. Then $G$ has a normal subgroup $H$ of finite index with $\left|H^{\prime}\right|<\infty$ and with $K^{t}[H]$ stably untwisted.

Proof. Without loss of generality, we can assume that $K$ is algebraically closed. Suppose $G$ has a subgroup $H$ of finite index with $\left|H^{\prime}\right|<\infty$ and with $K^{t}[H]$ stably untwisted. If $C$ is the core of $H$ in $G$, then we know that $C \triangleleft G, C \subseteq H$ and $|G: C|<\infty$. Furthermore, $C^{\prime} \subseteq H^{\prime}$ so $\left|C^{\prime}\right|<\infty$, and $K^{t}[C] \subseteq K^{t}[H]$ so $K^{t}[C]$ is stably untwisted, because untwistings involve a diagonal change of basis. In view of all of this, it suffices to prove the result without the normality conclusion. In particular, by the previous lemma, we can now assume that $K^{t}[G]$ satisfies an identity of the form

$$
\tilde{f}\left(\zeta_{1}, \zeta_{2}, \ldots, \zeta_{n}\right)=\sum_{\sigma \in \operatorname{Sym}_{n}} \alpha_{\sigma} \zeta_{\sigma(1)} \zeta_{\sigma(2)} \cdots \zeta_{\sigma(n)}
$$

with $\alpha_{\sigma} \in K^{t}[\mathfrak{G}]$ for all $\sigma \in \operatorname{Sym}_{n}$ and with $\alpha_{1} \neq 0$. Furthermore, we can suppose that $\left|G^{\prime}\right|<\infty$ and that $K^{t}\left[G^{\prime}\right]$ is central in $K^{t}[G]$.

We now procede to further modify $\tilde{f}\left(\zeta_{1}, \zeta_{2}, \ldots, \zeta_{n}\right)$. To start with, by multiplying $\tilde{f}$ on the left by $\bar{g}$ for some $g \in \mathfrak{G}$, we can assume that $1 \in \operatorname{supp} \alpha_{1}$. Next, if $x_{1}, x_{2}, \ldots, x_{n} \in G$, write $x=x_{1} x_{2} \cdots x_{n}$ and note that $\bar{x}_{\sigma(1)} \bar{x}_{\sigma(2)} \cdots \bar{x}_{\sigma(n)}=\beta_{\sigma} \bar{x}$ for some $\beta_{\sigma} \in K^{t}\left[G^{\prime}\right]$ depending upon $x_{1}, x_{2}, \ldots, x_{n}$. Thus

$$
0=\tilde{f}\left(\bar{x}_{1}, \bar{x}_{2}, \ldots, \bar{x}_{n}\right) \bar{x}^{-1}=\sum_{\sigma \in \operatorname{Sym}_{n}} \alpha_{\sigma} \beta_{\sigma}
$$

and

$$
0=\sum_{\sigma \in \operatorname{Sym}_{n}} \pi_{G^{\prime}}\left(\alpha_{\sigma} \beta_{\sigma}\right)=\sum_{\sigma \in \operatorname{Sym}_{n}} \pi_{G^{\prime}}\left(\alpha_{\sigma}\right) \beta_{\sigma}
$$

where $\pi_{G^{\prime}}: K^{t}[\mathfrak{G}] \rightarrow K^{t}\left[G^{\prime}\right]$ is the natural projection. In particular,

$$
0=\sum_{\sigma \in \operatorname{Sym}_{n}} \pi_{G^{\prime}}\left(\alpha_{\sigma}\right) \beta_{\sigma} \bar{x}=\sum_{\sigma \in \operatorname{Sym}_{n}} \pi_{G^{\prime}}\left(\alpha_{\sigma}\right) \bar{x}_{\sigma(1)} \bar{x}_{\sigma(2)} \cdots \bar{x}_{\sigma(n)},
$$

so we see that $K^{t}[G]$ satisfies

$$
\sum_{\sigma \in \operatorname{Sym}_{n}} \pi_{G^{\prime}}\left(\alpha_{\sigma}\right) \zeta_{\sigma(1)} \zeta_{\sigma(2)} \cdots \zeta_{\sigma(n)}
$$

Since $1 \in \operatorname{supp} \alpha_{1}$ implies that $\pi_{G^{\prime}}\left(\alpha_{1}\right) \neq 0$, we can now assume that $\alpha_{\sigma} \in K^{t}\left[G^{\prime}\right]$ for all $\sigma \in \operatorname{Sym}_{n}$. Note that $\Omega=\left\{\alpha_{\sigma} \mid \sigma \in \operatorname{Sym}_{n}\right\}$ is a nonzero, nonempty subset of the finite-dimensional commutative $K$-algebra $K^{t}\left[G^{\prime}\right]$ with $K$ algebraically closed. Thus, by Lemma 4.4, there exists $\gamma \in K^{t}\left[G^{\prime}\right]$ with $0 \neq \gamma \Omega \subseteq I$, a 1-dimensional ideal of $K^{t}\left[G^{\prime}\right]$. But $K^{t}[G]$ also satisfies $\gamma \tilde{f}\left(\zeta_{1}, \zeta_{2}, \ldots, \zeta_{n}\right)$, so by permuting the variables if necessary, we can now assume that all $\alpha_{\sigma} \in I$ and that $\alpha_{1} \neq 0$. In particular, we can write each $\alpha_{\sigma}=\alpha_{1} k_{\sigma}$ for some $k_{\sigma} \in K$, and consequently $K^{t}[G]$ satisfies

$$
\alpha_{1}\left(\sum_{\sigma \in \mathrm{Sym}_{n}} k_{\sigma} \zeta_{\sigma(1)} \zeta_{\sigma(2)} \cdots \zeta_{\sigma(n)}\right)
$$

with $k_{1}=1$.

Finally, let $J=\operatorname{ann}_{K^{t}\left[G^{\prime}\right]} \alpha_{1}=\operatorname{ann}_{K^{t}\left[G^{\prime}\right]} I$, so that $J$ is a maximal ideal of $K^{t}\left[G^{\prime}\right]$ with $K^{t}\left[G^{\prime}\right]=K+J$. Since $K^{t}[G]$ is free over $K^{t}\left[G^{\prime}\right]$, we then have $\operatorname{ann}_{K^{t}[G]} \alpha_{1}=$ 
$J \cdot K^{t}[G]$. By the above, we first see that $K^{t}[G] /\left(\operatorname{ann}_{K^{t}[G]} \alpha_{1}\right)$ satisfies the ordinary polynomial identity

$$
\sum_{\sigma \in \operatorname{Sym}_{n}} k_{\sigma} \zeta_{\sigma(1)} \zeta_{\sigma(2)} \cdots \zeta_{\sigma(n)}
$$

and second that

$$
K^{t}[G] /\left(\operatorname{ann}_{K^{t}[G]} \alpha_{1}\right)=K^{t}[G] /\left(J \cdot K^{t}[G]\right) \cong K^{t}\left[G / G^{\prime}\right]
$$

where the latter is a naturally obtained twisted group algebra of $G / G^{\prime}$. But then $K^{t}\left[G / G^{\prime}\right]$ satisfies an ordinary polynomial identity, so Lemma 4.3 implies that the abelian group $G / G^{\prime}$ has a subgroup $Z / G^{\prime}$ of finite index with $K^{t}\left[Z / G^{\prime}\right]=$ $K^{t}[Z] /\left(J \cdot K^{t}[Z]\right)$ commutative. In particular, $K^{t}[Z]$ has a commutative homomorphic image, and therefore it is stably untwisted by Lemma 4.1. Since $Z^{\prime} \subseteq G^{\prime}$, we have $\left|Z^{\prime}\right|<\infty$, and the result follows.

As an immediate consequence of the above and Lemma 4.2, we obtain

Corollary 4.7. The twisted group algebra $K^{t}[G]$ satisfies a nondegenerate multilinear generalized polynomial identity if and only if $G$ has a normal subgroup $H$ of finite index with $\left|H^{\prime}\right|<\infty$ and with $K^{t}[H]$ stably untwisted.

Finally, since untwisting and stable untwisting are inherited by subgroups, the preceding result yields a roundabout proof of the possibly surprising

Corollary 4.8. If $K^{t}[G]$ satisfies a nondegenerate multilinear generalized polynomial identity and if $H$ is a subgroup of $G$, then $K^{t}[H]$ also satisfies a nondegenerate multilinear generalized polynomial identity.

\section{REFERENCES}

[1] A. Giambruno, S. K. Sehgal and A. Valenti, Group algebras whose units satisfy a group identity, Proc. AMS 125 (1997), 629-634.

[2] I. M. Isaacs and D. S. Passman, Groups with representations of bounded degree, Canad. J. Math. 16 (1964), 299-309.

[3] Chia-Hsin Liu, Group algebras with units satisfying a group identity, Proc. AMS 127 (1999), $327-336$.

[4] Chia-Hsin Liu, Group identities on units of locally finite algebras and twisted group algebras, Commun. Algebra, to appear.

[5] D. S. Passman, Nil ideals in group rings, Mich. Math. J. 9 (1962), 375-384.

[6] D. S. Passman, Group rings satisfying a polynomial identity, J. Algebra 20 (1972), 103-117.

[7] D. S. Passman, Group rings satisfying a polynomial identity II, Pac. J. Math. 39 (1971). 425-438.

[8] D. S. Passman, The Algebraic Structure of Group Rings, Wiley-Interscience, New York, 1977.

[9] M. K. Smith Group algebras, J. Algebra 18 (1971), 477-499.

Mathematics Department, University of Wisconsin, Madison, Wisconsin 53706

E-mail address: passman@math.wisc.edu 